Supporting Information for

\title{
On the folding propensity of cyclohexylether- $\delta$-peptides
}

\author{
Hans-Dieter Arndt, ${ }^{\dagger, \ddagger}$ Burkhard Ziemer, ${ }^{\dagger}$ and Ulrich Koert ${ }^{*}$ \\ ${ }^{\dagger}$ Humboldt-Universität zu Berlin, Institut für Chemie, Brook-Taylor-Str. 2, D-12489 Berlin, Germany \\ ${ }^{\ddagger}$ New address: Max-Planck-Institut für molekulare Physiologie, Otto-Hahn-Str. 11, D-44227 Dortmund, Germany \\ *Philipps-Universität Marburg, Fachbereich Chemie, Hans-Meerwein-Str., D-35032 Marburg/Lahn, Germany
}

\section{Contents}

List of X-ray structures: S-1

General: S-2

Procedures: S-4

Additional Figures: S-18

References: S-19

\section{X-ray structural data}

All data for the X-ray structures reported in this publication have been deposited at the Cambridge Crystallographic Database as supplementary publications and can be retrieved on request (CCDC, 12 Union Road, Cambridge CB2 1EZ, England; Fax (+int) 1223-336-033; E-mail: deposit@ccdc.cam.ac.uk) by using the following access codes:

Methylated cyclotrimer 15 (Arndt2):

Benzyl-substituted cyclotrimer 24 (Arndt11):

Phenylethyl-lactam 18 (Arndt4):

Lactam 19 (Arndt6):
\#240797

\#240800

\#240798

\#240799

The X-ray structure of dimer 5 has been previously described: ${ }^{[1]}$ \#154390 


\section{General}

All reactions sensitive to air or moisture were conducted in flame-dried glassware under an atmosphere of dry argon. THF and $\mathrm{Et}_{2} \mathrm{O}$ were distilled from purple benzophenone-sodium. $\mathrm{CH}_{2} \mathrm{Cl}_{2}$, toluene, hexanes, pyridine, and $\mathrm{NEt}_{3}$ were distilled under $\mathrm{Ar}$ from $\mathrm{CaH}_{2}$. $\mathrm{MeOH}$ was distilled from $\mathrm{Mg}(\mathrm{OMe})_{2}$. Organolithium and amide base solutions were titrated against 2,2diphenyl-acetic acid. ${ }^{[2]}$ All starting materials and reagents were used as received unless noted otherwise. - PE: light petroleum, boiling range $40-60^{\circ} \mathrm{C}$. MTBE: Methyl-tert-butylether. - Thin layer chromatography (TLC) was performed on glass-supported Merck silica gel $60 \mathrm{~F}_{254}$ plates. Spots were visualized by UV light and by heat staining with $2 \%$ molybdophosphoric acid in ethanol. - Flash column chromatography (FCC) was performed on Merck silica gel 60 (40$63 \mu \mathrm{m})$. - Melting points were determined from pulverized material in glass capillaries and are uncorrected. - ${ }^{1} \mathrm{H}$ and ${ }^{13} \mathrm{C}$ NMR spectra were obtained on Bruker DPX 300 or AMX 600 spectrometers, respectively. All resonances are referenced to residual solvent signals. ${ }^{[3]}-$ IR: Perkin-Elmer FT-IR Spektrum 1600 or BioRad FT-IR 3000MX. - Optical rotations: PerkinElmer spektrophotopolarimeter 241, cuvette path length $10 \mathrm{~cm} . \mathrm{CHCl}_{3}$ for spectroscopy was filtered over basic aluminium oxide before use. - MS: Finigan MAT 95 (EI: 70 eV; FAB) or MSI Concept 1H (ESI). - CD-spectroscopy: JASCO 710, $\mathrm{N}_{2}$-flow 2 L/min, cylindrical cuvettes (2 mm path length). All spectra were FFT-smoothed means of 7 accumulations recorded at $20^{\circ} \mathrm{C}$. - Elemental analysis: Leco CHNS 932 Analysator (microanalytical facility, HU Berlin).

General Procedure \#1 (GP1): Azide and Z-group cleavage. The starting material was dissolved in $\mathrm{MeOH}$ (0.1 M), Pd/C (5\%, Degussa type E101 NO/W) was added (10 wt\%), and the mixture was degassed and hydrogenated (1 bar) with stirring until the conversion was complete. The catalyst was removed by filtration under argon over a pad of Celite and the pad was rinsed with $\mathrm{MeOH}$. The solvents were removed i.v., the residue was coevaporated with toluene/EtOH 10:1 and dried (0.1 mbar) to give the crude amine.

General Procedure \#2 (GP2): tert-Butylester cleavage. The starting material was dissolved in $\mathrm{CH}_{2} \mathrm{Cl}_{2}(0.5 \mathrm{M}$ or $1 \mathrm{~mL})$, cooled to $0^{\circ} \mathrm{C}$, and TFA was added (equal volume). After $15 \mathrm{~min}$ the cooling bath was removed, and the solution was stirred for $1.5 \mathrm{~h}$ at r.t. The volatiles were 
removed i.v., the residue coevaporated twice with toluene/EtOH 10:1, and dried (0.1 mbar) to give the crude carboxylic acid.

General procedure \#3 (GP3): Peptide coupling. The components were dissolved in $\mathrm{CH}_{2} \mathrm{Cl}_{2}$ $(0.1 \mathrm{M})$ and cooled to $0^{\circ} \mathrm{C}$. Then $\mathrm{HOBt}(1.5 \mathrm{eq})$ and $\mathrm{NEt}_{3}(1.5 \mathrm{eq})$ was added with stirring, followed after $15 \mathrm{~min}$ by EDC ( $1.25 \mathrm{eq}$ ). After $15 \mathrm{~min}$ at $0^{\circ} \mathrm{C}$, the mixture was stirred at r.t. until conversion was complete.

General procedure \#4 (GP4): Peptide cyclization. The $\omega$-Amino-carboxylic acid was dissolved in $\mathrm{CH}_{2} \mathrm{Cl}_{2} / \mathrm{DMF}(10: 1,2 \mathrm{mM})$ and added dropwise at constant rate to a stirred suspension of HATU (3 eq), HOAt (3 eq) and DIEA (6 eq) in $\mathrm{CH}_{2} \mathrm{Cl}_{2} / \mathrm{DMF}$ (10:1, equal volume) within $6 \mathrm{~h}$ (final conc.: $1 \mathrm{~mm}$ ) at r.t. The mixture was stirred for $12 \mathrm{~h}$, washed with sat. $\mathrm{NaHCO}_{3}$ solution, $1 \mathrm{M} \mathrm{NaHSO}_{4}$ solution, and brine ( $1 / 4$ vol. each), dried $\left(\mathrm{Na}_{2} \mathrm{SO}_{4}\right)$, and concentrated to give the crude cyclic peptide. 


\section{Synthetic Procedures}

(1S,2S)-2-Azido-1-(trimethylsilyloxy)cyclohexane (1). Azide 1 was prepared via slight modification of the procedures of Jacobsen et al.: ${ }^{[4,5]}(R, R)-S a l e n-\mathrm{CrN}_{3}$ (1.25 g, approx. $1.9 \mathrm{mmol}, 2 \mathrm{~mol} \%)^{[5]}$ were suspended in $\mathrm{Et}_{2} \mathrm{O}(50 \mathrm{~mL})$ and cooled to $0^{\circ} \mathrm{C}$. $\mathrm{i}$-PrOH $(1.5 \mathrm{~mL}$, approx. $20 \mathrm{~mol} \%$ ) were added. After $15 \mathrm{~min} \mathrm{TMSN}_{3}$ (13.3 mL, $\left.110 \mathrm{mmol}, 1.1 \mathrm{eq}\right)$ and cyclohexeneoxide $(9.9 \mathrm{~mL}, 100 \mathrm{mmol})$ were added and the mixture was stirred for $36 \mathrm{~h}$ at $4^{\circ} \mathrm{C}$. $\mathrm{Et}_{2} \mathrm{O}$ was removed i.v. and the residue was distilled into an ice-cooled receiver flask (Caution!) to yield azide 1 (20.2 g, 94\%, 93\% ee) as a colorless oil of unpleasant odor. The residual catalyst was reused several times without loss of activity. ${ }^{[4]} \mathrm{Bp}=43-45^{\circ} \mathrm{C}(0.06 \mathrm{mbar})$. TLC: $\mathrm{R}_{\mathrm{f}}=0.47$ (n-hexane/MTBE 20:1). GC: $t_{R}=29.7 \mathrm{~min}$ (50 m FS-Cyclodex alpha $-\mathrm{I} / \mathrm{P}, T_{c o l}=100^{\circ} \mathrm{C}, T_{i n j}=$ $200^{\circ} \mathrm{C}, T_{\text {det }}=250^{\circ} \mathrm{C}$, carrier $\left.\mathrm{H}_{2} 1.0 \mathrm{~kg} / \mathrm{cm}^{2}\right) ; t_{R}(R, R)=28.6 \mathrm{~min} .{ }^{1} \mathrm{H}-\mathrm{NMR}\left(300 \mathrm{MHz}, \mathrm{C}_{6} \mathrm{D}_{6}\right): \delta=$ 0.00 (s, 9H, OTMS), 0.51-1.69 (3 × m, 8H, 3-, 4-, 5-, 6- $\mathrm{H}_{2}$ ), 2.71-2.78 (m, 1H, 1-H), 3.11-3.19 (m, 1H, 2-H). ${ }^{13} \mathrm{C}-\mathrm{NMR}$ (75 MHz, $\mathrm{C}_{6} \mathrm{D}_{6}$ ): $\delta=0.0$ (OTMS), 23.7, 24.0 (C-4/C-5), 30.4, 34.7 (C3/C-6), 66.8 (C-2), 75.2 (C-1). IR (film): $v=$ 3332, 2942, 2865, 2505, 2099 (N N $_{3}$, 1451, 1304, 1253, 1103, 1012, 885, 843, $750 \mathrm{~cm}^{-1} \cdot[\alpha]^{20}{ }_{\mathrm{D}}=-28.6\left(c=2.89, \mathrm{CHCl}_{3}\right)$.

(1S,2S)-2-Azido-cyclohexanol (2). A solution of TMS-ether 1 (32.3 g, $151 \mathrm{mmol}$, 93\% ee) in $\mathrm{MeOH}(400 \mathrm{~mL})$ was treated with TFA $(50 \mu \mathrm{L})$ and stirred for $1 \mathrm{~h}$ at r.t. The volatiles were removed i.v. and FCC (250 g, PE/MTBE 5:1 $\rightarrow$ 3:1 $\rightarrow$ 2:1) followed by vacuum distillation (Caution!) provided alcohol 2 (20.5 g, $145 \mathrm{mmol}$, 96\%, 93\% ee) as a colorless oil of unpleasant odor, which solidified in the freezer. $\mathrm{Mp} 4-5^{\circ} \mathrm{C}$. Bp $44-46^{\circ} \mathrm{C}(0.2 \mathrm{mbar}) . n_{\mathrm{D}}^{20}=1.4955$. TLC: $R_{f}=0.22$ (n-hexane/EtOAc 5:1). ${ }^{1} \mathrm{H}-\mathrm{NMR}\left(300 \mathrm{MHz}, \mathrm{CDCl}_{3}\right): \delta=1.14-1.36$ (m, 4H, 3-, 4-, 5-, 6- $\left.\mathrm{H}_{2(\mathrm{ax})}\right)$, 1.62-1.79, 1.92-2.03 (2 × m, 4H, 3-, 4-, 5-, 6- $\left.\mathrm{H}_{2(\mathrm{eq})}\right), 2.72$ (d, J = $\left.3.2 \mathrm{~Hz}, 1 \mathrm{H}, \mathrm{OH}\right)$, 3.10-3.18 (m, 1H, 2-H), 3.30-3.37 (m, 1H, 1-H). ${ }^{13} \mathrm{C}-\mathrm{NMR}$ (75 MHz, $\left.\mathrm{CDCl}_{3}\right): \delta=23.7,24.1$ (C4/C-5), 29.7, 33.0 (C-3/C-6), 66.9 (C-2), 73.4 (C-1). IR (film): v= 3377, 2940, 2864, 2505, $2099\left(\mathrm{~N}_{3}\right), 1451,1259,1071,993,845,663 .[\alpha]_{\mathrm{D}}^{20}=+77.5\left(c=0.988, \mathrm{CHCl}_{3}, 93 \%\right.$ ee). Anal. calcd for $\mathrm{C}_{6} \mathrm{H}_{11} \mathrm{~N}_{3} \mathrm{O}$ (141.174): C, 51.05; H, 7.85; N, 29.77. Found: C, 51.15; H, 7.85; N, 30.04. 
(1’S,2’S)-(2'-Azido-cyclohexyl)oxy-acetic-acid-tert-butylester ( $\mathrm{N}_{2}=\mathrm{COA}$-tert-butylester, 3). Alcohol 2 (19.4 g, $137 \mathrm{mmol}, 90 \%$ ee) dissolved in toluene $(150 \mathrm{~mL})$ was treated at $0^{\circ} \mathrm{C}$ with tetrabutylammonium bromide (12 g, $37 \mathrm{mmol}, 0.27 \mathrm{eq})$, tert-butyl-bromoacetate (30 mL, $0.21 \mathrm{~mol}, 1.5 \mathrm{eq})$, and $12 \mathrm{M} \mathrm{NaOH}$ (100 mL, Caution!). The well-stirred mixture was warmed to r.t. in $18 \mathrm{~h}$, the layers were separated and the aqueous layer was extracted with $\mathrm{Et}_{2} \mathrm{O}(100 \mathrm{~mL})$. The combined org. layers were washed with $\mathrm{PO}_{4}{ }^{3-}$-buffer $(0.1 \mathrm{M}, \mathrm{pH} 7)$ and brine/ $\mathrm{H}_{2} \mathrm{O}$ 1:1 (200 mL), dried $\left(\mathrm{Na}_{2} \mathrm{SO}_{4}\right)$, and concentrated. The volatiles were removed i.v. $\left(0.1 \mathrm{mbar}, 60^{\circ} \mathrm{C}\right)$, and FCC (400 g, PE/MTBE 15:1 $\rightarrow$ 10:1) gave tert-butylester 3 (33.2 g, $130 \mathrm{mmol}$, 95\%) as a thick, colorless oil. TLC: $R_{f}=0.22$ (n-hexane/MTBE 12:1). ${ }^{1} \mathrm{H}$ NMR $\left(300 \mathrm{MHz}, \mathrm{CHCl}_{3}\right): \delta=$ 1.15-1.28 (m, 4 H, 3'-, 4'-, 5'-, 6'-H m, 4 H, 3'-, 4'-, 5'-, 6'-H2(eq)), 3.10-3.18 (m, 1 H, 1'-H), 3.27-3.35 (m, 1 H, 2'-H), 4.07 (s, 2 H,

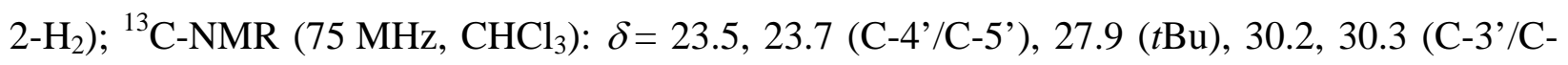
6’), 64.8 (C-2'), 67.7 (C-2), 81.4 (tBu), 82.0 (C-1'), 169.4 (C-1). IR (film): $v=$ 3477, 2939, 2866, 2505, $2100\left(-\mathrm{N}_{3}\right), 1748,1728(\mathrm{C}=\mathrm{O}), 1454,1260,1150,1125,844 .[\alpha]^{20}{ }_{\mathrm{D}}=-16.4(c=$ 1.03, $\mathrm{CHCl}_{3}, 90 \%$ ee). HRMS $\left(\mathrm{ESI}^{+},[\mathrm{M}+\mathrm{Na}]^{+}\right): \mathrm{m} / \mathrm{z}$ calcd 278.1473, found 278.1466. Anal. calcd for $\mathrm{C}_{12} \mathrm{H}_{21} \mathrm{~N}_{3} \mathrm{O}_{3}$ (255.32): C, 56.45; H, 8.29; N, 16.46. Found: C, 56.56; H, 7.99; N, 16.52.

\section{(1’S,2’S)-(2'-Azido-cyclohexyl)oxy-aceticacid-(2”,2”)-dimethylpropionicacid anhydride (4).}

Ester 3 (5.11 g, $20.0 \mathrm{mmol}$ ) was C-deprotected following GP2. The crude carboxylic acid thus obtained was dissolved in DMF (50 mL) and cooled to $0^{\circ} \mathrm{C}$. DIEA (3.6 mL, $21 \mathrm{mmol}, 1.2 \mathrm{eq}$ ) and pivalic chloride (2.46 mL, $20.0 \mathrm{mmol}, 1.1 \mathrm{eq}$ ) was added, and the mixture was stirred for $1 \mathrm{~h}$ at $0^{\circ} \mathrm{C}$. The mixed anhydride solution was generally used without further purification. The crude material could be stored at $-20^{\circ} \mathrm{C}$ after removal of DMF without significant loss of activity.

$\mathbf{N}_{2}=$ COA-COA-tert-butylester (5): Monomer $3(4.65 \mathrm{~g}, 18.2 \mathrm{mmol})$ was converted to the corresponding amine following GP1 (80 mL MeOH, $200 \mathrm{mg}$ Pd/C, 1 h, solvents removed at r.t.: 4.08 g, $17.8 \mathrm{mmol}, 98 \%$ ), dissolved in DMF (15 mL) and DIEA (3.6 mL, $21 \mathrm{mmol}, 1.2 \mathrm{eq})$, and added dropwise (5 min) to an ice-cooled solution of crude anhydride 4 (20 mmol) in DMF $(50 \mathrm{~mL})$. The mixture was stirred at $0^{\circ} \mathrm{C}$ for $1 \mathrm{~h}$, and for $5 \mathrm{~h}$ at r.t. Water $(150 \mathrm{~mL})$ and sat. $\mathrm{NaHCO}_{3}$ solution $(50 \mathrm{~mL})$ were added. The mixture was stirred for $2 \mathrm{~h}$ and extracted with MTBE $(3 \times 80 \mathrm{~mL})$. The combined org. layers were washed with water, $1 \mathrm{M} \mathrm{NaHSO}_{4}$, water, and 
brine consecutively (50 mL each), dried $\left(\mathrm{Na}_{2} \mathrm{SO}_{4}\right)$, and concentrated. FCC (150 g, PE/EtOAc 2:1 $\rightarrow$ 1:1 $\rightarrow$ 1:2) provided $5.08 \mathrm{~g}$ (12.4 mmol, 70\%) of dimer $\mathbf{5}$ as a colorless solid. Stereoisomers were removed by recrystallization from $n$-hexane/ $\mathrm{Et}_{2} \mathrm{O}\left(7: 1,25 \mathrm{~mL} ; 4^{\circ} \mathrm{C}\right.$, seed crystals). Mp 72$74^{\circ} \mathrm{C}$. TLC: $R_{f}=0.20$ ( $n$-hexane/EtOAc 1:1). ${ }^{1} \mathrm{H}$ NMR (300 MHz, $\left.\mathrm{CHCl}_{3}\right): \delta=1.05-1.38(\mathrm{~m}$,

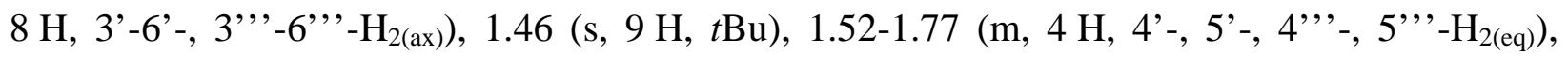

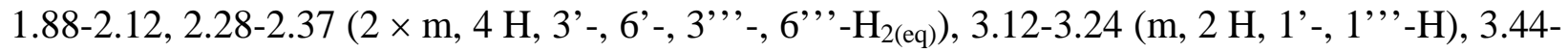
3.51 (m, 1 H, 2' ' '-H), 3.60-3.72 (m, 1 H, 2'-H), 3.94 (d, $J=17.0$ Hz, 1 H, 2-H $\mathrm{H}_{2 \mathrm{~A}}$ ), 3.97 (d, $J=$ $14.9 \mathrm{~Hz}, 1 \mathrm{H}, 2$ '”- $\mathrm{H}_{2 \mathrm{~A}}$ ), 4.01 (d, $J=17.0 \mathrm{~Hz}, 1 \mathrm{H}, 2-\mathrm{H}_{2 \mathrm{~B}}$ ), 4.09 (d, $J=14.9 \mathrm{~Hz}, 1 \mathrm{H}, 2$ '’ $-\mathrm{H}_{2 \mathrm{~B}}$ ), 7.64 (d, $J=6.0 \mathrm{~Hz}, 1 \mathrm{H}, \mathrm{NH}) .{ }^{13} \mathrm{C}-\mathrm{NMR}\left(75 \mathrm{MHz}, \mathrm{CHCl}_{3}\right): \delta=23.4,23.7,23.8,23.9$ (C-4', C5', C-4"', C-5'”'), 28.1 (tBu), 29.7, 29.8, 30.3, 30.8 (C-3', C-6', C-3'”, C-6’”), 53.0 (C-2'), 64.4 (C-2'”), 65.2 (C-2), 68.6 (C-2”'), 79.8 (C-1'), 81.4 (tBu), 82.1 (C-1’’'), 169.8, 170.4 (C=O); IR (KBr): $v=$ 3364, 2929, 2858, $2097\left(-\mathrm{N}_{3}\right), 1739$ (C=O), 1679, 1529, 1455, 1368, 1269, 1238, 1170, 1142, 1121, 842, 573. $[\alpha]^{20}{ }_{\mathrm{D}}=+64.2\left(c=1.00, \mathrm{CHCl}_{3}\right)$; HRMS $\left(\mathrm{EI},[\mathrm{M}+\mathrm{H}]^{+}\right): \mathrm{m} / \mathrm{z}$ calcd 411.2607, found 411.2609. Anal. calcd for $\mathrm{C}_{20} \mathrm{H}_{34} \mathrm{~N}_{4} \mathrm{O}_{5}$ (410.52): C, 58.52; H, 8.35; N, 13.65. Found: C, 58.80; H, 8.22; N, 13.75.

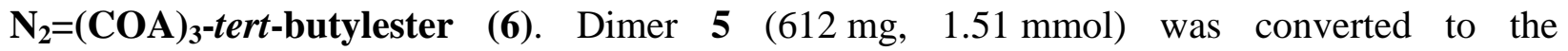
corresponding amine following GP1 and coupled with crude anhydride 4 (2.40 mmol, 1.6 eq) as described for dimer 5. FCC $(2 \times 40$ g, hexanes/i-PrOH 9:1 $\rightarrow$ 8:1 $\rightarrow$ 7:2) gave trimer 6 (775 mg, $1.37 \mathrm{mmol}, 91 \%$ ) as a sticky colorless gum, which was isomerically pure by TLC and ${ }^{1} \mathrm{H}-\mathrm{NMR}$. TLC: $R_{f}=0.22$ ( $n$-hexane/i-PrOH 7:1), minor diastereomer: $R_{f}=0.33 .{ }^{1} \mathrm{H}$ NMR $(300 \mathrm{MHz}$,

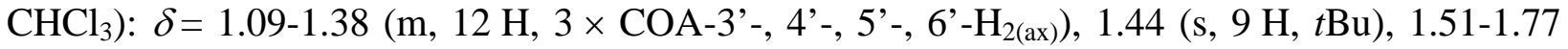
(m, 6 H, 3 × COA-4'-, 5'- $\left.\mathrm{H}_{2(\mathrm{eq})}\right), 1.94-2.10$ (m, 6 H, 3 × COA-3'-, 6'- $\left.\mathrm{H}_{2(\mathrm{eq})}\right), 2.80-3.12$ (m, $2 \mathrm{H}$, ${ }^{1-2} \mathrm{COA}-1$ '-H), 3.12-3.21 (m, $1 \mathrm{H},{ }^{3} \mathrm{COA}-1$ '-H), 3.29 (dt, $\left.J=10.3,4.5 \mathrm{~Hz}, 1 \mathrm{H},{ }^{1} \mathrm{COA}-2{ }^{\prime}-\mathrm{H}\right)$, 3.57-3.66 (m, $1 \mathrm{H},{ }^{3} \mathrm{COA}-2$ '-H), 3.79-3.88 (m, $1 \mathrm{H},{ }^{2} \mathrm{COA}-2$ '-H), 3.86 (d, $J=14.7 \mathrm{~Hz}, 1 \mathrm{H}$, COA-2- $\mathrm{H}_{2 \mathrm{~A}}$ ), 3.87 (d, $J=15.2 \mathrm{~Hz}, 1 \mathrm{H}, \mathrm{COA}-2-\mathrm{H}_{2 \mathrm{~A}}$ ), 3.98 (s, $\left.2 \mathrm{H},{ }^{2} \mathrm{COA}-2-\mathrm{H}_{2}\right), 4.04$ (d, $J=$ $14.7 \mathrm{~Hz}, 1 \mathrm{H}, \mathrm{COA}-2-\mathrm{H}_{2 \mathrm{~B}}$ ), 4.05 (d, $\left.J=15.2 \mathrm{~Hz}, 1 \mathrm{H}, \mathrm{COA}-2-\mathrm{H}_{2 \mathrm{~B}}\right), 6.95$ (d, $J=8.4 \mathrm{~Hz}, 1 \mathrm{H}$, ${ }^{2} \mathrm{COA}-\mathrm{NH}$ ), 7.26 (d, $\left.J=6.8 \mathrm{~Hz}, 1 \mathrm{H},{ }^{3} \mathrm{COA}-\mathrm{NH}\right) .{ }^{13} \mathrm{C}-\mathrm{NMR}\left(75 \mathrm{MHz}, \mathrm{CHCl}_{3}\right): \delta=23.4,23.5$, 23.7, 23.8, 23.9, 24.0 (3 × each COA-C-4', C-5'), 28.1 ( $t \mathrm{Bu}$ ), 29.8, 29.9, 30.0, 30.3, 30.8, 30.9 (3 × each COA-C3', C-6'), 52.1, $52.6\left({ }^{2-3} \mathrm{COA}-\mathrm{C}-2^{\prime}\right), 64.7$ ( $\left.{ }^{1} \mathrm{COA}-\mathrm{C}-2^{\prime}\right), 66.0$ ( $\left.{ }^{3} \mathrm{COA}-\mathrm{C}-2\right), 68.1$, 
$68.5\left({ }^{1-2} \mathrm{COA}-\mathrm{C}-2\right), 80.1$ ( ${ }^{3} \mathrm{COA}-\mathrm{C}-1$ '), 81.4 (tBu), 81.7, $82.5\left({ }^{1} \mathrm{COA}-,{ }^{2} \mathrm{COA}-\mathrm{C}-1\right.$ ') $)$ 169.5, 169.9, 170.3 (C=O). IR (Film): $v=3410$, 3334, 2977, 2940, 2862, 2099 (-N 3$), 1747$, 1682, 1651, 1532, 1454, 1368, 1245, 1126, 846, 734, 582. $[\alpha]^{20}{ }_{\mathrm{D}}=+64.9\left(c=0.288, \mathrm{CH}_{2} \mathrm{Cl}_{2}\right)$. HRMS (EI, $\left.[\mathrm{M}+\mathrm{H}]^{+}\right): \mathrm{m} / \mathrm{z}$ calcd 565.3554 , found 565.3555 .

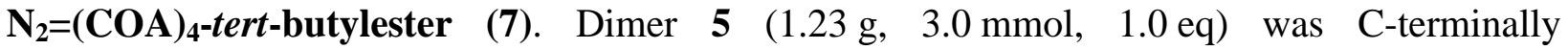
deprotected following GP2 and independently another batch of dimer 5 (1.23 g, $3.0 \mathrm{mmol})$ was N-terminally deprotected. Both batches were combined and coupled using GP3. FCC (50 g, cyclohexane/acetone 2:1 $\rightarrow$ 3:2 $\rightarrow$ 1:1) provided tetramer 7 (2.13 g, $2.95 \mathrm{mmol}, 98 \%$ ) as a white foam. TLC: $R_{f}=0.09$ (cyclohexane/acetone 2:1). ${ }^{1} \mathrm{H}-\mathrm{NMR}$ (300 MHz, $\left.\mathrm{CDCl}_{3}\right) ; \delta=1.12-1.37$ (m, 16H, 4 × COA-3'-, 4'-, 5'-, 6'- $\left.\mathrm{H}_{2(\mathrm{ax})}\right), 1.44$ (s, 9H, tBu), 1.53-1.76 (m, 8H, 4 × COA-4'-, 5'$\left.\mathrm{H}_{2(\mathrm{eq})}\right), 1.86-2.10$ (m, 8H, $\left.4 \times \mathrm{COA}^{3} 3^{\prime}-, 6^{\prime}-\mathrm{H}_{2(\mathrm{eq})}\right), 3.06,3.09$ (2 $\times$ m, 2H, $2 \times \mathrm{COA}-1$ '-H), 3.18 (app. td, $J=9.4,3.8 \mathrm{~Hz}, 1 \mathrm{H}, \mathrm{COA}-1^{\prime}-\mathrm{H}$ ) 3.26 (app. td, $J=9.3,3.6 \mathrm{~Hz}, 1 \mathrm{H}, \mathrm{COA}-1^{\prime}-\mathrm{H}$ ), 3.36 (app. td, $J=9.4,4.5 \mathrm{~Hz}, 1 \mathrm{H},{ }^{1} \mathrm{COA}-2$ '-H), 3.67-3.76 (m, $1 \mathrm{H},{ }^{4} \mathrm{COA}-2$ '-H), 3.75 (d, $J=14.5 \mathrm{~Hz}$, $1 \mathrm{H}, \mathrm{COA}-2-\mathrm{H}_{2}$ ), 3.82-3.89 (m, 2H, ${ }^{2-3} \mathrm{COA}-2$ '-H), 3.88 (d, $J=15.6 \mathrm{~Hz}, 1 \mathrm{H}, \mathrm{COA}-2-\mathrm{H}_{2}$ ), 3.89 (d, $\left.J=14.7 \mathrm{~Hz}, 1 \mathrm{H}, \mathrm{COA}-2-\mathrm{H}_{2}\right), 4.01$ (s, 2H, COA-2- $\left.\mathrm{H}_{2}\right), 4.03$ (d, $J=15.6 \mathrm{~Hz}, 1 \mathrm{H}, \mathrm{COA}-2-\mathrm{H}_{2}$ ), 4.06 (d, $J=14.5 \mathrm{~Hz}, 1 \mathrm{H}, \mathrm{COA}-2-\mathrm{H}_{2}$ ), 4.08 (d, $\left.J=14.7 \mathrm{~Hz}, 1 \mathrm{H}, \mathrm{COA}-2-\mathrm{H}_{2}\right), 6.97$ (d, $J=8.6 \mathrm{~Hz}$, 1H, NH), 7.01 (d, $J=8.3 \mathrm{~Hz}, 1 \mathrm{H}, \mathrm{NH}$ ), 7.25 (d, $\left.J=7.0 \mathrm{~Hz}, 1 \mathrm{H},{ }^{4} \mathrm{COA}-\mathrm{NH}\right) .{ }^{13} \mathrm{C}-\mathrm{NMR}$ (75 MHz, $\mathrm{CDCl}_{3}$ ); $\delta=23.4,23.5,23.6,23.8,23.9,24.2$, 24.3 (4 × each COA-C-4', C-5'), $28.1(t \mathrm{Bu}), 29.9$, 30.0, 30.3, 30.7, 30.8, 30.9, 31.1 (4 × each COA-C3', C-6'), 51.8, 52.1, $52.2\left({ }^{2-4} \mathrm{COA}-\mathrm{C}-2^{\prime}\right), 64.7$ ( ${ }^{1} \mathrm{COA}-\mathrm{C}-2$ '), 66.3 ( $\left.{ }^{4} \mathrm{COA}-\mathrm{C}-2\right), 68.0,68.2,68.4,\left({ }^{1-3} \mathrm{COA}-\mathrm{C}-2\right), 80.3\left({ }^{4} \mathrm{COA}-\mathrm{C}-1{ }^{\prime}\right), 81.3(\mathrm{Bu})$, 81.8, 82.5, $82.6\left({ }^{1-3} \mathrm{COA}-\mathrm{C}-1\right.$ '), 169.6, 169.7, 170.0, 170.3 (C=O). IR (film): $v=3402$, 3325, 3056, 2942, 2860, $2096\left(\mathrm{~N}_{3}\right)$, 1747, 1682, 1645, 1557, 1520, 1455, 1368, 1260, 1153, 1039, 846, 734, 581. $[\alpha]^{20}{ }_{D}=+65.2\left(c=0.790, \mathrm{CH}_{2} \mathrm{Cl}_{2}\right)$. HRMS (EI, $\left.[\mathrm{M}]^{+}\right): \mathrm{m} / \mathrm{z}$ calcd 720.4422, found 720.4415 .

$\mathbf{N}_{2}=(\mathbf{C O A})_{6}$-tert-butylester (8). Tetramer 7 (361 mg, $500 \mu \mathrm{mol}$, $1.0 \mathrm{eq}$ ) was C-terminally deprotected following GP2 and dimer 5 (205 mg, $500 \mu \mathrm{mol}$ ) were N-terminally deprotected following GP1. Both batches were combined and coupled following GP3. FCC (60 g, EtOAc/MeOH 20:1 $\rightarrow$ 15:1 $\rightarrow$ 10:1 $\rightarrow \mathrm{CH}_{2} \mathrm{Cl}_{2} / \mathrm{MeOH}$ 94:6) provided hexamer 8 (493 mg 
(478 $\mu \mathrm{mol}, 96 \%)$ as a colorless gum. TLC: $R_{f}=0.26\left(\mathrm{CH}_{2} \mathrm{Cl}_{2} / \mathrm{MeOH} 20: 1\right) .{ }^{1} \mathrm{H}-\mathrm{NMR}(600 \mathrm{MHz}$, $\left.\mathrm{CDCl}_{3}\right) ; \delta=1.14-1.41\left(\mathrm{~m}, 24 \mathrm{H}, 6 \times \mathrm{COA}^{2}\right.$ 3'-, 4' $^{\prime}, 5^{\prime}-$, 6' $\left.^{\prime} \mathrm{H}_{2(\mathrm{ax})}\right), 1.44$ (s, 9H, $\left.t \mathrm{Bu}\right), 1.55-1.82(\mathrm{~m}$, $\left.12 \mathrm{H}, 6 \times \mathrm{COA}^{\prime} 4^{\prime}-, 5^{\prime}-\mathrm{H}_{2(\mathrm{eq})}\right), 1.82-2.15$ (m, 12H, $6 \times$ COA-3'-, 6'- $\left.\mathrm{H}_{2(\mathrm{eq})}\right), 3.03$ (dt, $J=9.8$, $4.1 \mathrm{~Hz}, 1 \mathrm{H}, \mathrm{COA}-1$ '-H), 3.06-3.13 (m, 3H, $3 \times \mathrm{COA}-1$ '-H), 3.16 (dt, $J=9.2,3.8 \mathrm{~Hz}, 1 \mathrm{H}, \mathrm{COA}-$ 1'-H), 3.26 (dt, $\left.J=3.6,9.0 \mathrm{~Hz}, 1 \mathrm{H}, \mathrm{COA}-1^{\prime}-\mathrm{H}\right), 3.35$ (m, 1H, $\left.{ }^{1} \mathrm{COA}-2^{\prime}-\mathrm{H}\right), 3.67-3.91$ (m, 5H, ${ }^{2-6} \mathrm{COA}-2{ }^{\prime}-\mathrm{H}$ ), 3.72 (d, $\left.J=14.8 \mathrm{~Hz}, 1 \mathrm{H}, \mathrm{COA}-2-\mathrm{H}_{2}\right), 3.75$ (d, $J=14.3 \mathrm{~Hz}, 1 \mathrm{H}, \mathrm{COA}-2-\mathrm{H}_{2}$ ), 3.77 (d, $J=15.3 \mathrm{~Hz}, 1 \mathrm{H}, \mathrm{COA}-2-\mathrm{H}_{2}$ ), 3.88 (d, $J=14.5 \mathrm{~Hz}, 1 \mathrm{H}, \mathrm{COA}-2-\mathrm{H}_{2}$ ), 3.89 (d, $J=15.9 \mathrm{~Hz}, 1 \mathrm{H}$, COA-2- $\mathrm{H}_{2}$ ), 4.00-4.12 (m, 7H, COA-2- $\mathrm{H}_{2}$ ), 6.92 (d, $\left.J=8.9 \mathrm{~Hz}, 1 \mathrm{H}, \mathrm{NH}\right), 6.93$ (d, $J=9.4 \mathrm{~Hz}$, 1H, NH), 6.94 (d, $J=9.7 \mathrm{~Hz}, 1 \mathrm{H}, \mathrm{NH}), 6.98$ (d, $J=8.8 \mathrm{~Hz}, 1 \mathrm{H}, \mathrm{NH}), 7.21$ (d, $J=7.7 \mathrm{~Hz}, 1 \mathrm{H}$, $\left.{ }^{6} \mathrm{COA}-\mathrm{NH}\right) .{ }^{13} \mathrm{C}-\mathrm{NMR}\left(75 \mathrm{MHz}, \mathrm{CDCl}_{3}\right)$; $\delta=23.5,23.6,23.7,23.9,24.2,24.3,24.4,24.4$ ( COA-C-4', C-5') 28.0 (tBu), 29.9, 30.0, 30.3, 30.7, 30.9, 31.0, 31.1 (6 × COA-C3', C-6'), 51.6, 51.7, 51.8, 52.0, 52.1 ( ${ }^{2-6} \mathrm{COA}-\mathrm{C}-2$ ') 64.7 ( ${ }^{1} \mathrm{COA}-\mathrm{C}-2$ '), 66.6 ( $\left.{ }^{6} \mathrm{COA}-\mathrm{C}-2\right), 67.9,68.0,68.1$, 68.2, $68.6\left({ }^{1-5} \mathrm{COA}-\mathrm{C}-2\right), 80.3\left({ }^{6} \mathrm{COA}-\mathrm{C}-1\right.$ ') $81.1(t \mathrm{Bu}), 81.8,82.5,82.6,83.0\left({ }^{1-5} \mathrm{COA}-\mathrm{C}-1\right.$ '), 169.8, 169.8, 169.9, 169.9, 170.0, 170.2 (C=O). IR(film): $v=3392,3323,3056,2936,2861$, $2097\left(N_{3}\right), 1744,1668,1539,1454,1368,1262,1143,1038,846,734,581 .[\alpha]^{20}=+69.3$ (0.992, $\mathrm{CH}_{2} \mathrm{Cl}_{2}$ ). HRMS (FAB, [M] $\left.{ }^{+}\right): \mathrm{m} / \mathrm{z}$ calcd 1030.6314, found 1030.6329 .

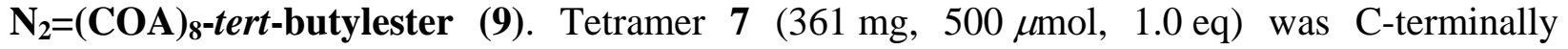
deprotected following GP2 and independently another batch of tetramer 7 (361 mg, $500 \mu \mathrm{mol}$ ) were N-terminally deprotected. Both batches were combined and coupled following GP3. FCC (60 g, $\mathrm{CH}_{2} \mathrm{Cl}_{2} /$ Aceton 1:1 $\rightarrow \mathrm{CH}_{2} \mathrm{Cl}_{2} / \mathrm{MeOH}$ 95:5 $\rightarrow$ 93:7) gave octamer 9 (650 mg, $484 \mu \mathrm{mol}$, 97\%) as a colorless foam. TLC: $R_{f}=0.25\left(\mathrm{CH}_{2} \mathrm{Cl}_{2} /\right.$ acetone 1:2). ${ }^{1} \mathrm{H}-\mathrm{NMR}\left(300 \mathrm{MHz}, \mathrm{CDCl}_{3}\right)$;

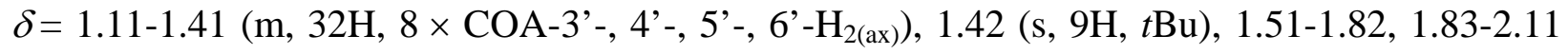
(2 m, 32H, $8 \times$ COA-3'-, 4'-, 5', 6'- $\left.\mathrm{H}_{2(\mathrm{eq})}\right), 2.99-3.19\left(\mathrm{~m}, 7 \mathrm{H}, 7 \times \mathrm{COA}-1^{\prime}-\mathrm{H}\right), 3.24(\mathrm{~m}, 1 \mathrm{H}$, COA-1'-H), 3.33 (m, 1H, ${ }^{1} \mathrm{COA}-2$ '-H), 3.64-3.90 (m, 14H, ${ }^{2-8} \mathrm{COA}-2$ '- $\mathrm{H}, \mathrm{COA}-2-\mathrm{H}_{2}$ ), 3.95-4.08 (m, 9H, COA-2- $\mathrm{H}_{2}$ ), 6.92 (app. d, $J=9.1 \mathrm{~Hz}, 3 \mathrm{H}, \mathrm{NH}$ ), 6.94 (d, $J=8.3 \mathrm{~Hz}, 1 \mathrm{H}, \mathrm{NH}$ ), 6.95 (d, $J=8.3 \mathrm{~Hz}, 1 \mathrm{H}, \mathrm{NH}), 6.97$ (d, $J=8.7 \mathrm{~Hz}, 1 \mathrm{H}, \mathrm{NH}), 7.18$ (d, $\left.J=7.5 \mathrm{~Hz}, 1 \mathrm{H},{ }^{8} \mathrm{COA}-\mathrm{NH}\right) .{ }^{13} \mathrm{C}-$ NMR (75 MHz, $\mathrm{CDCl}_{3}$ ); $\delta$ = 23.5, 23.6, 23.7, 23.9, 24.2, 24.4, 24.4, 24.5 (COA-C-4', C-5') 28.1 (tBu), 29.9, 30.0, 30.3, 30.7, 30.9, 31.0, 31.1 (COA-C3', C-6'), 51.6, 51.7, 51.8, 52.0, $52.1\left(^{2-}\right.$ ${ }^{8} \mathrm{COA}-\mathrm{C}-2$ ') 64.7 ( ${ }^{1} \mathrm{COA}-\mathrm{C}-2$ '), $66.7\left({ }^{8} \mathrm{COA}-\mathrm{C}-2\right), 67.9,68.0,68.1,68.1,68.2,68.6\left({ }^{1-7} \mathrm{COA}-\mathrm{C}-\right.$ 
2), 80.3 ( ${ }^{8} \mathrm{COA}-\mathrm{C}-1$ '), 81.1 (tBu), 81.8, 82.5, 82.7, $83.0\left({ }^{1-7} \mathrm{COA}-\mathrm{C}-1\right.$ ') $, 169.8,169.9,169.9$, 170.0, 170.2 (C=O). IR (film): $v=$ 3390, 3322, 3054, 2936, 2860, $2099\left(\mathrm{~N}_{3}\right)$, 1747, 1682, 1652, 1539, 1454, 1263, 1142, 1123, 846, 734, 581. $[\alpha]^{20}{ }_{\mathrm{D}}=+67.6\left(c=1.19, \mathrm{CH}_{2} \mathrm{Cl}_{2}\right)$. HRMS $(\mathrm{FAB}$, $\left.[\mathrm{M}+\mathrm{H}]^{+}\right): \mathrm{m} / \mathrm{z}$ calcd 1341.8285 , found 1341.8239 .

$\mathbf{N}_{2}=(\mathbf{C O A})_{12}$-tert-butylester (10). Hexamer 8 (49.6 mg, $48.1 \mu \mathrm{mol}$, $\left.1.0 \mathrm{eq}\right)$ were C-terminally deprotected following GP2 and independently another batch of hexamer 8 (49.6 mg, $48.1 \mu \mathrm{mol}$ ) were N-terminally deprotected following GP1. Both batches were combined and coupled following GP3 (5 mL DMF, $3 \mathrm{~h})$. FCC (10 g, $\left.\mathrm{CHCl}_{3} / \mathrm{MeOH} 100: 3 \rightarrow 100: 4\right)$ delivered dodecamer 10 (84.8 mg, $43.2 \mu \mathrm{mol}, 90 \%)$ as a colorless resin. TLC: $R_{f}=0.31\left(\mathrm{CHCl}_{3} / \mathrm{MeOH}\right.$ 100:4). ${ }^{1} \mathrm{H}-\mathrm{NMR}$ (300 MHz, $\left.\mathrm{CDCl}_{3}\right) ; \delta=1.11-1.42$ (m, 48H, $12 \times \mathrm{COA}^{2}{ }^{\prime}-, 4^{\prime}-, 5^{\prime}-, 6^{\prime}-\mathrm{H}_{2(\mathrm{ax})}$ ),

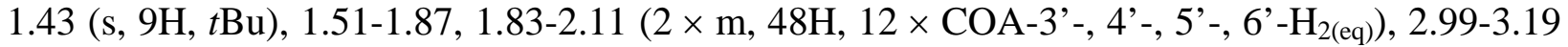
(m, $\left.11 \mathrm{H}, 11 \times \mathrm{COA}-1^{\prime}-\mathrm{H}\right), 3.21-3.39\left(\mathrm{~m}, 2 \mathrm{H}, \mathrm{COA}-1^{\prime}-\mathrm{H},{ }^{1} \mathrm{COA}-2^{\prime}-\mathrm{H}\right), 3.65-3.90\left(\mathrm{~m}, 22 \mathrm{H},{ }^{2-}\right.$ ${ }^{12} \mathrm{COA}-2$ '-H, COA-2- $\left.\mathrm{H}_{2}\right)$, 3.95-4.08 (m, 13H, COA-2- $\left.\mathrm{H}_{2}\right), 6.89-6.99\left(\mathrm{~m}, 10 \mathrm{H},{ }^{2-11} \mathrm{COA}-\mathrm{NH}\right)$, 7.19 (d, $\left.J=7.5 \mathrm{~Hz}, 1 \mathrm{H},{ }^{12} \mathrm{COA}-\mathrm{NH}\right) .{ }^{13} \mathrm{C}-\mathrm{NMR}\left(75 \mathrm{MHz}, \mathrm{CDCl}_{3}\right) ; \delta=23.5,23.6,23.7,23.9$, 24.2, 24.4, 24.5 (12 × COA-C-4', C-5'), 28.1 (tBu), 29.9, 30.0, 30.0, 30.1, 30.3, 30.7, 30.9, 31.0, 31.1 (12 × COA-C3', C-6'), 51.7, 51.8, 52.0, 52.1 ( ${ }^{2-12} \mathrm{COA}-\mathrm{C}-2$ '), 64.7 ('COA-C-2'), 66.7 $\left.\left({ }^{12} \mathrm{COA}-\mathrm{C}-2\right), 67.9,68.0,68.1,68.1,68.2,68.6\left({ }^{1-11} \mathrm{COA}-\mathrm{C}-2\right), 80.4\left({ }^{12} \mathrm{COA}-\mathrm{C}-1\right)\right), 81.1(t \mathrm{Bu})$, 81.9, 82.5, 82.7, 83.1 ( (-11 COA-C-1'), 169.8, 169.9, 169.9, 169.9, 170.0, 170.1, 170.1, 170.2 $(\mathrm{C}=\mathrm{O})$. MS (ESI, $\left.[\mathrm{M}+\mathrm{Na}]^{+}\right): \mathrm{m} / \mathrm{z}$ calcd 1984.2, found 1983.8 .

Cyclo(-COA-COA-COA-) (11). Trimer 6 (148 mg, $262 \mu \mathrm{mol})$ was consecutively C-terminally (GP2) and N-terminally deprotected. The crude aminoacid was cyclized following GP4. Triple FCC $\left(15 \mathrm{~g}, 10 \mathrm{~g}, 3 \mathrm{~g}, \mathrm{CHCl}_{3} / \mathrm{EtOH}\right.$ 99:1 $\rightarrow$ 97:3 $\rightarrow$ 95:5 $\rightarrow$ 92:8) gave the cyclic trimer $\mathbf{1 1}$ (86.4 mg, $186 \mu \mathrm{mol}, 71 \%$ ) as a colorless solid of low solubility (exception: mixtures of $\mathrm{CHCl}_{3}$ with alcohols or DMSO). Mp $>260^{\circ} \mathrm{C}$. TLC: $R_{f}=0.22\left(\mathrm{CHCl}_{3} / \mathrm{EtOH} 95: 5\right) .{ }^{1} \mathrm{H}-\mathrm{NMR}(300 \mathrm{MHz}$, $\mathrm{CDCl}_{3} / d_{4}-\mathrm{MeOH}$ 20:1): $\delta=1.15-1.35\left(\mathrm{~m}, 6 \mathrm{H}, 4^{\prime}-5^{\prime}-\mathrm{H}_{2(\mathrm{ax})}\right), 1.35$ (m, 3H, 3'- $\left.\mathrm{H}_{2(\mathrm{ax})}\right), 1.59(\mathrm{~m}$, 3H, 6'- $\left.\mathrm{H}_{2(\mathrm{ax})}\right), 1.70\left(\mathrm{~m}, 3 \mathrm{H}, 5^{\prime}-\mathrm{H}_{2(\mathrm{eq})}\right), 1.79\left(\mathrm{~m}, 3 \mathrm{H}, 4{ }^{\prime}-\mathrm{H}_{2(\mathrm{eq})}\right), 1.98\left(\mathrm{~m}, 3 \mathrm{H}, 6^{\prime}-\mathrm{H}_{2(\mathrm{eq})}\right), 2.05(\mathrm{~m}$, 3H, 3'- $\left.\mathrm{H}_{2(\mathrm{eq})}\right), 3.51$ (m, 3H, 1'-H), 3.62 (m, 3H, 2'-H), 3.79 (d, J = $14.3 \mathrm{~Hz}, 3 \mathrm{H}, 2-\mathrm{H}_{2 \mathrm{~A}}$ ), 3.99 (d, $\left.J=14.3 \mathrm{~Hz}, 3 \mathrm{H}, 2-\mathrm{H}_{2 \mathrm{~B}}\right), 7.03(\mathrm{~d}, J=7.1 \mathrm{~Hz}, 3 \mathrm{H}, \mathrm{NH}) .{ }^{13} \mathrm{C}-\mathrm{NMR}\left(75 \mathrm{MHz}, \mathrm{CDCl}_{3} / d_{4}-\mathrm{MeOH}\right.$ 
20:1): $\delta=24.1,24.5$ (C-4', C-5'), 30.0, 30.7 (C-3’, C-6'), 53.3 (C-2'), 65.4 (C-2), 78.5 (C-1'), $170.2(\mathrm{C}-1)$. IR (KBr): $v=3405,3288,2934,2858,1664,1544,1450,1151,1114,601 .[\alpha]_{\mathrm{D}}=$ $+77\left(c=0.32, \mathrm{CH}_{2} \mathrm{Cl}_{2} / \mathrm{MeOH} 1: 1\right)$. HRMS(EI, [M] $\left.{ }^{+}\right): \mathrm{m} / \mathrm{z}$ calcd 465.2839, found 465.2837.

Cyclo(-COA-COA-COA-COA-) (12). Tetramer 7 (163 mg, $226 \mu \mathrm{mol})$ was consecutively Cterminally (GP2) and N-terminally deprotected. The crude aminoacid was cyclized following GP4. FCC (20 g, EtOAc/MeOH 100:6 $\rightarrow$ 100:8 $\rightarrow$ 10:1 $\rightarrow$ 100:12) gave cyclic tetramer 12 (100 mg, $161 \mu \mathrm{mol}, 71 \%$ ) as a colorless solid, which crystallized from $\mathrm{CHCl}_{3} /$ toluene in fine, solvent-containing needles. $\mathrm{Mp}>260^{\circ} \mathrm{C}\left(\mathrm{CHCl}_{3}\right)$. TLC: $R_{f}=0.14\left(\right.$ EtOAc/MeOH 200:15). ${ }^{1} \mathrm{H}-$ NMR (300 MHz, $\left.\mathrm{CDCl}_{3}\right) ; \delta=1.15-1.26$ (m, 12H, 3'-, 4'-, 5'- $\left.\mathrm{H}_{2(\mathrm{ax})}\right), 1.44-1.55$ (m, 4H, 6'- $\mathrm{H}_{2(\mathrm{ax})}$ ), 1.70-1.76, 2.00-2.14 (2 × m, 16H, 3'-, 4'-, 5'-, 6'- $\left.\mathrm{H}_{2(\mathrm{eq})}\right)$, 3.46-3.63 (m, 8H, 1'-, 2'-H), 3.78 (d, $J=14.9 \mathrm{~Hz}, 4 \mathrm{H}, 2-\mathrm{H}_{2 \mathrm{~A}}$ ), 4.05 (d, $\left.J=14.9 \mathrm{~Hz}, 4 \mathrm{H}, 2-\mathrm{H}_{2 \mathrm{~B}}\right), 7.11$ (d, $\left.J=6.4 \mathrm{~Hz}, 4 \mathrm{H}, \mathrm{NH}\right) .{ }^{13} \mathrm{C}-$ NMR (75 MHz, CDCl 3 ); $\delta=$ 24.1, 24.8 (C-4', C-5'), 30.6, 31.1 (C-3', C-6'), 54.6 (C-2'), 67.2 (C-2), 80.6 (C-1'), 170.6 (C-1). IR (KBr): $v=3404,3067$, 2933, 2859, 1661, 1542, 1450, 1384 , 1338, 1246, 1120, 848, 559.). $[\alpha]^{27}{ }_{D}=109\left(c=0.598, \mathrm{CH}_{2} \mathrm{Cl}_{2} / \mathrm{MeOH} 1: 1\right)$. HRMS (EI, $\left.[\mathrm{M}]^{+}\right)$: $\mathrm{m} / \mathrm{z}$ calcd 620.3786, found 620.3786. Anal. calcd for $\mathrm{C}_{193} \mathrm{H}_{315} \mathrm{~N}_{24} \mathrm{O}_{49} \mathrm{Cl}_{3}$ (3862.14, (12) $\times$ $\mathrm{CHCl}_{3} \times \mathrm{H}_{2} 0$ ): C, 60.02; H, 8.22; N, 8.70; Cl, 2.75. Found: C, 59.93; H, 8.19; N, 8.60; Cl, 2.86.

Cyclo(-COA-COA-COA-COA-COA-COA-) (13). Hexamer 8 (292 mg, $283 \mu \mathrm{mol})$ was consecutively C-terminally (GP2) and N-terminally deprotected. The crude aminoacid was cyclized following GP4. Double FCC (40 g each, $\mathrm{CHCl}_{3} / \mathrm{MeOH}$ 200:6 $\rightarrow$ 200:7 $\rightarrow$ 200:8) gave cyclic hexamer $13(140 \mathrm{mg}, 150 \mu \mathrm{mol}, 53 \%)$ as a colorless solid of low solubility. TLC: $R_{f}=$ $0.15\left(\mathrm{CH}_{2} \mathrm{Cl}_{2} / \mathrm{MeOH}\right.$ 97:3). ${ }^{1} \mathrm{H}-\mathrm{NMR}\left(300 \mathrm{MHz}, \mathrm{CDCl}_{3}\right): \delta=1.18-1.39$ (m, 24H, 3'-, 4'-, 5'-, 6'$\left.\mathrm{H}_{2(\mathrm{ax})}\right)$, 1.67-1.77, 1.93-2.03 (2 × m, 24H, 3'-, 4'-, 5'-, 6'- $\left.\mathrm{H}_{2(\mathrm{eq})}\right), 3.23-3.30$ (m, 6H, 1'-H), 3.443.87 (m, 6H, 2'-H), 3.85 (d, $J=15.1$ Hz, 6H, 2- $\mathrm{H}_{2}$ ), 4.04 (d, $J=15.1 \mathrm{~Hz}, 6 \mathrm{H}, 2-\mathrm{H}_{2}$ ), 6.87 (d, $J=$ $8.7 \mathrm{~Hz}, 6 \mathrm{H}, \mathrm{NH}) .{ }^{13} \mathrm{C}-\mathrm{NMR}$ (75 MHz, $\mathrm{CDCl}_{3}$ ): $\delta=24.0,24.4$ (C-4', C-5'), 30.7, 31.3 (C-3', C6'), 52.5 (C-2'), 68.3 (C-2), 81.9 (C-1'), 170.1 (C-1). IR (KBr): $v=3406$, 2932, 2855, 1646, $1538,1449,1343,1321,1261,1148,1104,1014,616 .[\alpha]_{\mathrm{D}}=+72.1\left(c=1.06, \mathrm{CH}_{2} \mathrm{Cl}_{2} / \mathrm{MeOH}\right.$ 1:1). HRMS (EI, [M] $\left.{ }^{+}\right): \mathrm{m} / \mathrm{z}$ calcd 930.5678 , found 930.5671. 
Cyclo(-COA-COA-COA-COA-COA-COA-COA-COA-) (14). Octamer 9 (304 mg, $227 \mu \mathrm{mol})$ was consecutively C-terminally (GP2) and N-terminally deprotected. The crude aminoacid was cyclized following GP4. Triple FCC (40 g, 30 g, 15 g, $\mathrm{CHCl}_{3} / \mathrm{MeOH}$ 200:7 $\rightarrow$ 200:8) provided cyclic octamer $14(126 \mathrm{mg}, 101 \mu \mathrm{mol}, 45 \%)$ as a colorless wax. TLC: $R_{f}=0.27\left(\mathrm{CH}_{2} \mathrm{Cl}_{2} / \mathrm{MeOH}\right.$ 20:1). ${ }^{1} \mathrm{H}-\mathrm{NMR}\left(300 \mathrm{MHz}, \mathrm{CDCl}_{3}\right.$ ): $\delta=1.14-1.33$ (m, 32H, 3'-, 4'-, 5'-, 6'- $\mathrm{H}_{2(\mathrm{ax})}$ ), 1.65-1.73, 1.90-2.03 (2 × m, 32H, 3'-, 4'-, 5'-, 6'-H2(eq)), 3.13-3.19 (m, 8H, 1'-H), 3.70-3.86 (m, 8H, 2'-H), 3.84 (d, $J=14.8 \mathrm{~Hz}, 8 \mathrm{H}, 2-\mathrm{H}_{2}$ ), 4.00 (d, $J=14.8 \mathrm{~Hz}, 8 \mathrm{H}, 2-\mathrm{H}_{2}$ ), 6.93 (d, $\left.J=8.5 \mathrm{~Hz}, 8 \mathrm{H}, \mathrm{NH}\right)$. ${ }^{13} \mathrm{C}-\mathrm{NMR}$ (75 MHz, $\mathrm{CDCl}_{3}$ ): $\delta=$ 23.7, 24.2 (C-4', C-5'), 30.2, 31.2 (C-3', C-6'), 52.2 (C-2'), 68.5 (C-2), 82.2 (C-1'), 170.0 (C-1). IR (film): $v=3304,2934,2860,1667,1540,1450,1341$, 1265, 1151, 1120, 734, 572. $[\alpha]_{\mathrm{D}}=+61.5\left(c=1.31, \mathrm{CH}_{2} \mathrm{Cl}_{2} / \mathrm{MeOH}\right.$ 1:1). HRMS (FAB, $\left.[\mathrm{M}+\mathrm{H}]^{+}\right): \mathrm{m} / \mathrm{z}$ calcd 1241.7649 , found 1241.7691 .

$N^{1}, N^{2}, N^{3}$-Trimethyl-cyclo(-COA-COA-COA-) (15). Cyclic trimer 11 (45.7 mg, $\left.98.2 \mu \mathrm{mol}\right)$ in DMF (1 mL) was treated with $\mathrm{NaH}$ (60\% in paraffin, $0.5 \mathrm{mmol}, 5 \mathrm{eq})$, MeI $(0.1 \mathrm{~mL}, 1.6 \mathrm{mmol}$, $16 \mathrm{eq})$ was added and stirred for $14 \mathrm{~h}$. HOAc $(0.1 \mathrm{~mL})$ was added at $0^{\circ} \mathrm{C}$, the mixture was concentrated i.v. and the residue was partitioned between $\mathrm{CHCl}_{3}$ and halfsat. brine (10 $\mathrm{mL}$ each). The org. layer was extracted with $\mathrm{CHCl}_{3}(2 \times 10 \mathrm{~mL})$, the combined org. layers were washed with brine $(10 \mathrm{~mL})$, dried $\left(\mathrm{Na}_{2} \mathrm{SO}_{4}\right)$, and concentrated. FCC $\left(5 \mathrm{~g}, \mathrm{CHCl}_{3} / \mathrm{EtOH}\right.$ 95:5 $\rightarrow$ 93:7) gave the trimethylated trimer 15 (43.0 mg, $84.7 \mu \mathrm{mol}, 86 \%)$ as a colorless solid, which was crystallized from acetone. Mp $168.5-170^{\circ} \mathrm{C}$. TLC: $R_{f}=0.13\left(\mathrm{CHCl}_{3} / \mathrm{EtOH} 20: 1\right) .{ }^{1} \mathrm{H}-\mathrm{NMR}$ (300 MHz, $\left.\mathrm{CDCl}_{3}\right)$ : several conformers, $\delta=1.08-1.70,1.90-2.35$ (2 × m, 24H, 3'-, 4'-, 5'-, 6'$\mathrm{H}_{2}$ ), 2.70-2.83 (m, 9H, N-Me), 3.12-3.37 (m, 4H, 1'-H, 2'-H), 3.58-4.44 (m, 8H, 2'-H, 2- $\mathrm{H}_{2}$ ). ${ }^{13} \mathrm{C}-\mathrm{NMR}\left(75 \mathrm{MHz}, \mathrm{CDCl}_{3}\right.$ ): several conformers, $\delta=24.1,24.4,24.5,24.6,24.7,24.8,24.9$ (C4’, C-5'), 26.9, 27.0, 27.1, 28.6, 29.7, 30.0, 30.6, 30.9, 32.1 (C-3’, C-6', N-Me), 59.2, 59.5 (C2'), 68.4, 69.3, 70.7 (C-2), 75.0, 76.6, 77.7, 77.8 (C-1'), 168.3, 168.5, 168.9, 169.4, 170.0, 171.5 $(\mathrm{C}-1) .[\alpha]_{D}^{20}=24.1\left(c=0.453, \mathrm{CH}_{2} \mathrm{Cl}_{2}\right)$. HRMS $\left(\mathrm{EI},[\mathrm{M}]^{+}\right): \mathrm{m} / \mathrm{z}$ calcd 507.3308, found 507.3310.

(1S,2S,1'S)-N-2''-Chloracetyl-2-(1'-phenyl)-ethyl-aminocyclohexanol (17). (1,2-trans,1'S)-2(1'-phenyl-ethyl)-amino-cyclohexanol (13.8 g, $63 \mathrm{mmol}, 1: 1 \mathrm{mixture}$ of diastereomers, prepared 
from (S)-1-phenylethylamine and cyclohexeneoxide in $97 \%$ yield ${ }^{[6]}$ in $\mathrm{CH}_{2} \mathrm{Cl}_{2}(150 \mathrm{~mL})$ and $\mathrm{NEt}_{3}\left(26 \mathrm{~mL}, 0.19 \mathrm{~mol}, 3 \mathrm{eq}\right.$ ) was cooled to $0^{\circ} \mathrm{C}$. Cloroaceticacid chloride $(5.5 \mathrm{~mL}, 69 \mathrm{mmol}$, $1.1 \mathrm{eq})$ in $\mathrm{CH}_{2} \mathrm{Cl}_{2}(30 \mathrm{~mL})$ was added dropwise $(15 \mathrm{~min})$ with concomitant darkening of the mixture. After stirring for $12 \mathrm{~h}$ at r.t. saturated $\mathrm{NaHCO}_{3}(100 \mathrm{~mL})$ was added, the mixture was diluted with MTBE (200 mL) and filtered over a pad of Celite. The layers were separated, and the org. layer was washed with $1 \mathrm{M} \mathrm{H}_{2} \mathrm{SO}_{4}$, water, and brine (100 mL each), dried $\left(\mathrm{Na}_{2} \mathrm{SO}_{4}\right)$, and concentrated. FCC (300g, PE/EtOAc 2:1 $\rightarrow$ 1:1 $\rightarrow$ EtOAc) of the solid residue gave (1S,2S)diastereomer 17 (4.48 g, $15.1 \mathrm{mmol}$, 24\%) followed by the more polar (1R,2R)-diastereomer 17a (6.32 g, $21.4 \mathrm{mmol}, 34 \%$ ), both as colorless solids. 17 crystallized nicely from $n$-hexane/toluene $(2: 1,10 \mathrm{~mL} / \mathrm{g})$ whereas the 17a was crystallized from $n$-hexane/EtOAc $(2: 1,10 \mathrm{~mL} / \mathrm{g})$. Data for 17: Mp 125.5 - 126.2 ${ }^{\circ} \mathrm{C}$. TLC: $R_{f}=0.33$ (n-hexane/EtOAc 1:1). ${ }^{1} \mathrm{H}-\mathrm{NMR}\left(300 \mathrm{MHz}, \mathrm{CDCl}_{3}\right)$ : $\delta=0.99-2.01$ (m, 11H, 3-, 4-, 5-, 6- $\mathrm{H}_{2}, 2$ '- $\mathrm{H}_{3}$ ), 3.31-4.43 (m, 5H, 1-, 2-, 1'-H, 2'’- $\mathrm{H}_{2}$ ), 5.02 (br s, 1H, OH), 7.11-7.49 (m 5H, Ph). ${ }^{13} \mathrm{C}-\mathrm{NMR}\left(75 \mathrm{MHz}, \mathrm{CDCl}_{3}\right.$ ): $\delta=167.0$ (C-1’'), 141.9, 129.0, 128.8, 128.4, 127.6, 127.2 (Ph), 69.8 (C-1), 65.4 (C-1'), 54.2 (C-2), 43.4 (C-2’'), 34.2, 29.9 (C-3, C-6), 25.3, 24.2 (C-4, C-5), 19.1 (C-2'). IR (KBr): $v=3395,2936,2858,1693,1447,1374$, 1322, 1175, 1084, 766, 714, 694, 616, 600. $[\alpha]^{20}{ }_{D}=+3.3\left(c=0.30, \mathrm{CH}_{2} \mathrm{Cl}_{2}\right)$. Anal Calcd. for $\mathrm{C}_{16} \mathrm{H}_{22} \mathrm{NO}_{2} \mathrm{Cl}$ (295.811): C, 64.97; H, 7.50; N, 4.74, Cl, 11.99. Found: C, 64.93; H, 7.65; N, 4.73; Cl, 12.05. Data for 17a: Mp $145.5-146^{\circ} \mathrm{C}$. TLC: $R_{f}=0.15$ (n-hexane/EtOAc 1:1). ${ }^{1} \mathrm{H}-$ NMR (300 MHz, $\mathrm{CDCl}_{3}$ ): $\delta=0.53-2.29$ (m, 11H, 3-, 4-, 5-, 6- $\left.\mathrm{H}_{2}, 2^{\prime}-\mathrm{H}_{3}\right), 3.40-4.52$ (m, 5H, 1-,

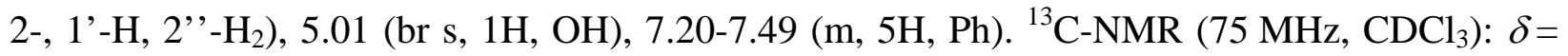
167.2 (C-1'”), 141.6, 128.6, 128.1, 128.0, 126.4, 126.1 (Ph), 70.0 (C-1), 64.8 (C-1'), 53.0 (C-2), 43.1 (C-2”'), 35.3, 29.9 (C-3, C-6), 25.4, 24.4 (C-4, C-5), 18.9 (C-2'). IR (KBr): $n=3436,2951$, 2930, 1641, 1454, 1440, 1372, 1256, 1236, 1070, 791, 698, 610, 581. $[\alpha]_{D}^{20}=-34.8(c=1.98$, $\mathrm{CH}_{2} \mathrm{Cl}_{2}$ ). Anal. calcd for $\mathrm{C}_{16} \mathrm{H}_{22} \mathrm{NO}_{2} \mathrm{Cl}$ (295.811): C, 64.97; H, 7.50; N, 4.74; Cl, 11.99. Found: C, 65.25; H, 7.54; N, 4.73; Cl, 12.11.

(4aS,8aS,1'S)- $N$-(1'Phenyl)-ethyl-4-oxa-perhydrochinolin-2-on (18). Alcohol 17 (4.26 g, $14.4 \mathrm{mmol})$ in $\mathrm{THF}(80 \mathrm{~mL})$ was treated at $0^{\circ} \mathrm{C}$ with $\mathrm{KOtBu}(1.9 \mathrm{~g}, 17 \mathrm{mmol}, 1.2 \mathrm{eq})$ with stirring. After 45 min acetic acid $(1 \mathrm{~mL})$ was added, the mixture was partitioned between MTBE and brine $\left(100 \mathrm{~mL}\right.$ each). The org. layer was dried $\left(\mathrm{MgSO}_{4}\right)$ and concentrated. FCC (100g, PE/MTBE 50:50 $\rightarrow$ 40:60) and crystallization from $n$-hexane gave ether 18 (3.42 g, $13.2 \mathrm{mmol}$, 
92\%) as clear, colorless blocks. X-ray crystallography confirmed the stereochemical assignment. Mp 85-85.6 ${ }^{\circ} \mathrm{C}$. TLC: $R_{f}=0.28$ ( $n$-hexane/MTBE 1:1). ${ }^{1} \mathrm{H}$-NMR $\left(300 \mathrm{MHz}, \mathrm{CDCl}_{3}\right): \delta=0.72-$ 0.85, 1.01-1.36, 1.45-1.52 (3 × m, 6H, 5-, 6-, 7-, 8- $\mathrm{H}_{2}$ ), 1.62 (d, $J=7.2 \mathrm{~Hz}, 3 \mathrm{H}, 2$ '- $\mathrm{H}_{3}$ ), 1.85-1.96 (m, 2H, 5-, 8- $\mathrm{H}_{2}$ ), 2.93-3.01 (m, 1H, 8a-H), 3.21-3.29 (m, 1H, 4a-H), 4.21 (d, $J=16.6$ Hz, 1H, 3$\mathrm{H}_{2}$ ), 4.32 (d, $J=16.6 \mathrm{~Hz}, 1 \mathrm{H}, 3-\mathrm{H}_{2}$ ), 5.62 (q, $\left.J=7.2 \mathrm{~Hz}, 1 \mathrm{H}, 1^{\prime}-\mathrm{H}\right), 7.14-7.29$ (m, 5H, Ph). ${ }^{13} \mathrm{C}-$ NMR (75 MHz, $\left.\mathrm{CDCl}_{3}\right): \delta=168.2$ (C-2), 139.3, 128.3, 128.3, 127.3, 127.3, 126.9 (Ph), 78.6 (C4a), 69.0 (C-3), 59.7 (C-1'), 51.8 (C-8a), 30.8, 30.0 (C-5, C-8), 23.9, 23.8 (C-6, C-7), 18.6 (C2'). IR (KBr): $v=3442,2941,2864,1635,1421,1350,1289,1117,703 .[\alpha]^{20}{ }_{D}=+55.9(c=$ 0.984, $\mathrm{CHCl}_{3}$ ). Anal. calcd for $\mathrm{C}_{16} \mathrm{H}_{21} \mathrm{NO}_{2}$ (259.350): C, 74.10; $\mathrm{H}, 8.16 ; \mathrm{N}, 5.40$. Found: C, 74.21; H, 8.22; N, 5.39 .

(4aS,8aS)-4-Oxa-perhydrochinolin-2-on (19). $N$-(Phenyl)ethylamid 18 (65 mg, $0.25 \mathrm{mmol}$ ) in formic acid $(99 \%, 1 \mathrm{~mL})$ was heated to $100^{\circ} \mathrm{C}$ for $8 \mathrm{~h}$ in a sealed tube. The volatiles were removed and FCC $\left(2 \mathrm{~g}, \mathrm{CH}_{2} \mathrm{Cl}_{2} / \mathrm{MeOH}\right.$ 99:1 $\rightarrow$ 95:5) gave lactam $19(23.1 \mathrm{mg}, 0.15 \mathrm{mmol}$, $60 \%)$ as a colorless solid, which was characterized by X-ray crystallography. Mp $170-170.5^{\circ} \mathrm{C}$. TLC: $R_{f}=0.16\left(\mathrm{CH}_{2} \mathrm{Cl}_{2} / \mathrm{MeOH} 30: 1\right) .{ }^{1} \mathrm{H}-\mathrm{NMR}\left(300 \mathrm{MHz}, \mathrm{CDCl}_{3}\right): \delta=1.21-1.39$ (m, 4H, 5-, 6-, 7-, 8- $\left.\mathrm{H}_{2(\mathrm{ax})}\right), 1.62-2.02$ (m, 4H, 5-, 6-, 7-, 8- $\left.\mathrm{H}_{2(\mathrm{eq})}\right)$, 3.07-3.24 (m, 2H, 4a-, 8a-H), 4.18-4.31 (m, 2H, 3-H $\mathrm{H}_{2}$ ), 7.31 (m, 1H, NH). ${ }^{13} \mathrm{C}-\mathrm{NMR}\left(75 \mathrm{MHz}, \mathrm{CDCl}_{3}\right.$ ): $\delta=169.7$ (C-2), 78.0 (C-4a), 67.9 (C-3), 55.9 (C-8a), 30.7, 29.8 (C-5, C-8), 24.1, 23.4 (C-6, C-7). IR (KBr): $v=3440$, 3173, 3075, 2937, 2863, 1699, 1644, 1416, 1380, 1360, 1109, 796. $[\alpha]^{25}=+47.2\left(c=0.252, \mathrm{CHCl}_{3}\right)$. Anal. calcd for $\mathrm{C}_{8} \mathrm{H}_{13} \mathrm{NO}_{2}$ (155.129): C, 61.91; H, 8.44; N, 9.03. Found: C, 61.87; H, 8.41; N, 9.11.

Lactam 19 via the azide route. Azide 1 (214 mg, $1.0 \mathrm{mmol}$, 93\% ee) in $\mathrm{MeOH}(10 \mathrm{~mL})$ was treated with TFA (10 $\mu \mathrm{L})$ and stirred for $30 \mathrm{~min}$ at r.t. 5\% Pt/C (20 mg) was added, and the stirred mixture was hydrogenated ( $1 \mathrm{bar}$ ) for $3 \mathrm{~d}$. The suspension was filtered through a pad of Celite and concentrated to dryness. The crude (1S,2S)-2-aminocyclohexanol was dissolved in $\mathrm{CH}_{2} \mathrm{Cl}_{2}(10 \mathrm{~mL}), \mathrm{NEt}_{3}(1 \mathrm{~mL}, 1.0 \mathrm{M}$ in toluene, $1.0 \mathrm{eq})$ was added and the mixture was cooled to $0^{\circ} \mathrm{C}$. 2-Chloroacetic acid anhydride $(180 \mathrm{mg}, 1.05 \mathrm{mmol}, 1.05 \mathrm{eq})$ was added with vigorous stirring and the cooling bath was removed. After $30 \mathrm{~min}$ gab $1 \mathrm{M} \mathrm{NaHSO}_{4}(2 \mathrm{~mL})$ and halfsat. brine $(10 \mathrm{~mL})$ were added. The layers were separated and the aqueous layer was extracted with EtOAc/i-PrOH (10:1, $4 \times 10 \mathrm{~mL})$. The combined org. Layers were washed with brine $(10 \mathrm{~mL})$, 
dried $\left(\mathrm{Na}_{2} \mathrm{SO}_{4}\right)$, and concentrated. Recrystallization from hot toluene gave 2-chloroaceticacid(2'-hydroxy)-cyclohexylamide 19a (188 mg, $0.98 \mathrm{mmol}$, 98\%) as colorless needles. Mp 140$141^{\circ} \mathrm{C}$. TLC: $R_{f}=0.11(\mathrm{MTBE}) .{ }^{1} \mathrm{H}-\mathrm{NMR}\left(300 \mathrm{MHz}, \mathrm{CDCl}_{3}\right): \delta=1.10-1.35,1.61-2.01(2 \times \mathrm{m}$, 8H, 3-, 4-, 5-, 6- $\mathrm{H}_{2}$ ), 3.01 (br s, 1H, OH), 3.20-3.35, 3.51-3.66 (2 × m, 2H, 1-, 2-H), 3.94-4.09 (m, 2H, 2'- $\mathrm{H}_{2}$ ), 6.42 (br s, 1H, NH). ${ }^{13} \mathrm{C}-\mathrm{NMR}\left(75 \mathrm{MHz}, \mathrm{CDCl}_{3}\right.$ ): $\delta=167.2$ (C-1'), 74.6 (C-1), 56.0 (C-2), 42.6 (C-2'), 34.4, 31.2 (C-3, C-6), 24.4, 24.0 (C-4, C-5). IR (KBr): v= 3307, 2932, 1645, 1548, 1409, 1264, 1060, 1046, 771, 579. $[\alpha]^{20}{ }_{\mathrm{D}}=-6.5\left(c=0.617, \mathrm{CHCl}_{3}\right.$. Anal Calcd. for $\mathrm{C}_{8} \mathrm{H}_{14} \mathrm{NO}_{2} \mathrm{Cl}$ (191.659): C, 50.13; H, 7.36; N, 7.31; Cl, 18.50. Found: C, 49.83; H, 7.22; N, 7.05; $\mathrm{Cl}, 18.10$.

Alcohol 19a (55.0 mg, $287 \mu \mathrm{mol})$ was reacted with $\mathrm{KOtBu}$ as described for 18. Extraction with $\mathrm{CHCl}_{3}$, FCC (4 g, $\mathrm{CH}_{2} \mathrm{Cl}_{2} /$ Aceton 5:2 $\rightarrow$ 2:1), and crystallization (n-hexane/toluene 2:1) provided lactam 19 (33.2 mg, $214 \mu \mathrm{mol}, 75 \%, 93 \%$ ee) as a colorless solid, Mp 168-170 ${ }^{\circ} \mathrm{C}$. $[\alpha]_{D}^{25}=+43.3\left(c=0.254, \mathrm{CHCl}_{3} ; 93 \%\right.$ ee). The substance was otherwise identical to the material described above.

(4aS,8aS)- $N$-Carboxybenzyl-4-oxa-perhydrochinolin-2-on $\quad$ (20). Lactam 19 (180 mg, $1.16 \mathrm{mmol})$ in THF $(13 \mathrm{~mL})$ were cooled to $-78^{\circ} \mathrm{C}$ and $0.80 \mathrm{~mL} n \mathrm{BuLi}(1.6 \mathrm{M}$ in hexanes, $1.3 \mathrm{mmol}, 1.1 \mathrm{eq}$ ) were added dropwise and the mixture was stirred for $15 \mathrm{~min}$. ZCl (175 $\mu \mathrm{L}$, $1.24 \mathrm{mmol}, 1.07 \mathrm{eq}$ ) was added dropwise, and the mixture was stirred for $30 \mathrm{~min}$ at $-78^{\circ} \mathrm{C}$ and for $30 \mathrm{~min}$ at $0^{\circ} \mathrm{C}$. The mixture was partitioned between MTBE and sat. $\mathrm{NH}_{4} \mathrm{Cl}(20 \mathrm{~mL}$ each) and the aq. layer was extracted with MTBE $(2 \times 10 \mathrm{~mL})$. The combined org. layers were washed with brine, dried $\left(\mathrm{Na}_{2} \mathrm{SO}_{4}\right)$, and concentrated. FCC (16 g, $n$-hexane/EtOAc 5:1 $\rightarrow$ 3:1) gave N-Zlactam 20 (266 mg, $888 \mu \mathrm{mol}, 77 \%$ ) as a colorless resin. TLC: $R_{f}=0.28$ ( $n$-hexane/EtOAc 3:1). ${ }^{1} \mathrm{H}-\mathrm{NMR}\left(300 \mathrm{MHz}, \mathrm{CDCl}_{3}\right.$ ): $\delta=0.95-1.49$ (m, 4H, 5-, 6-, 7-, 8- $\mathrm{H}_{2(\mathrm{ax})}$ ), 1.63-1.77 (m, 2H, 6-, 7$\left.\mathrm{H}_{2(\mathrm{eq})}\right)$, 1.95-2.05 (m, 1H, 5- $\left.\mathrm{H}_{2(\mathrm{eq})}\right)$, 2.12-2.20 (m, 1H, 8- $\left.\mathrm{H}_{2(\mathrm{eq})}\right)$, 3.10-3.29 (m, $\left.1 \mathrm{H}, 4 \mathrm{a}-\mathrm{H}\right), 3.55-$ 3.69 (m, 1H, 8a-H), 4.07 (d, $J=15 \mathrm{~Hz}, 1 \mathrm{H}, 3-\mathrm{H}_{2}$ ), 4.23 (d, $J=15 \mathrm{~Hz}, 1 \mathrm{H}, 3-\mathrm{H}_{2}$ ), 5.23 (s, 2H, Z), 7.16-7.39 (m, 5H, Ph). ${ }^{13} \mathrm{C}-\mathrm{NMR}\left(75 \mathrm{MHz}, \mathrm{CDCl}_{3}\right.$ ): $\delta=167.7$ (C-2), 152.7 (Z), 134.8, 128.6, 128.5, 128.2 (Ph), 78.3 (C-4a), 69.6 (C-3), 69.0 (O-CH $-\mathrm{Ph}), 60.7$ (C-8a), 30.8, 29.4 (C-5, C-8), 24.1, 23.7 (C-6, C-7). 
(3R,4aS,8aS)- $N$-Carboxybenzyl-3-benzyl-4-oxa-perhydrochinolin-2-on (21). HMDS (42 $\mu \mathrm{L}$, $203 \mu \mathrm{mol}, 1.01 \mathrm{eq})$ in THF (3 mL) were treated at $0^{\circ} \mathrm{C}$ with $n$-BuLi $(125 \mu \mathrm{L}, 1.6 \mathrm{M}$ in hexanes, $200 \mu \mathrm{mol})$. The mixture was stirred for $15 \mathrm{~min}$ and cooled to $-78^{\circ} \mathrm{C}$ gekühlt. Lactam 20 (57.9 $\mathrm{mg}$ (200 $\mu \mathrm{mol})$ in THF $(1.6 \mathrm{~mL})$ was added dropwise $(1 \mathrm{~min})$. After $30 \mathrm{~min} \mathrm{BnBr}(40 \mu \mathrm{L}, 0.30$ mmol, $1.5 \mathrm{eq}$ ) was added and the mixture was stirred for $6 \mathrm{~h}$ at $-78^{\circ} \mathrm{C}$. Then more $\mathrm{BnBr}$ was added (40 $\mu \mathrm{L}, 0.30 \mathrm{mmol}, 1.5 \mathrm{eq}$ ) and the mixture was warmed to $0^{\circ} \mathrm{C}$ within $12 \mathrm{~h}$. The mixture was diluted with $\mathrm{MTBE}$ and sat. $\mathrm{NH}_{4} \mathrm{Cl}$ (10 mL each), stirred for $1 \mathrm{~h}$ at r.t., and the layers were separated. The aq. layer was extracted with MTBE $(2 \times 10 \mathrm{~mL})$, washed with brine $(10 \mathrm{~mL})$, dried $\left(\mathrm{Na}_{2} \mathrm{SO}_{4}\right)$, and concentrated. FCC (10 g, $n$-hexane/EtOAc 5:1) provided lactam 21 (23.5 mg, $61.9 \mu \mathrm{mol}, 31 \%$, dr = 149:1 by HPLC) as a colorless oil as well as unreacted starting material (4.4 mg, $15 \mu \mathrm{mol}, 8 \%$ ). TLC: $R_{f}=0.19$ ( $n$-hexane/EtOAc 5:1). HPLC: $t_{R}=9.8 \mathrm{~min}$ (Supersphere Si60, $n$-hexane/i-PrOH 99:1, $1 \mathrm{~mL} / \mathrm{min}, 220 \mathrm{~nm}$ ); (3S)-isomer: $t_{R}=8.4 \mathrm{~min} .{ }^{1} \mathrm{H}$ NMR (300 MHz, $\left.\mathrm{CDCl}_{3}\right): \delta=1.03$ (m, 1H, 8- $\mathrm{H}_{2(\mathrm{ax})}$ ), 1.18-1.33 (m, 2H, 6-, 7- $\mathrm{H}_{2(\mathrm{ax})}$ ), 1.43 (m,

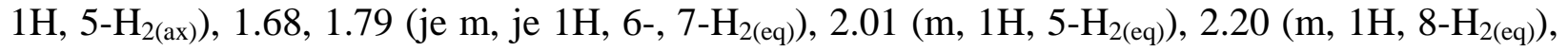
3.05 (dd, $J=7.1,14.3 \mathrm{~Hz}, 1 \mathrm{H}, 1$ '- $\mathrm{H}_{2 \mathrm{~A}}$ ), 3.29 (dd, $J=3.4,14.3 \mathrm{~Hz}, 1 \mathrm{H}, 1$ '- $\mathrm{H}_{2 \mathrm{~B}}$ ), 3.31 (m, 1H, 4aH), 3.45 (dt, $J=3.4,9.8$ Hz, 1H, 8a-H), 4.46 (dd, $J=3.4,7.0$ Hz, 1H, 3-H), 5.21 (s, 2H, Z), 7.167.26, 7.28-7.39 (je m, je 5H, $2 \times \mathrm{Ph}) .{ }^{13} \mathrm{C}-\mathrm{NMR}$ (75 MHz, $\mathrm{CDCl}_{3}$ ): $\delta=170.3(\mathrm{C}-2), 152.5(\mathrm{Z})$, 136.9, 135.0, 129.8, 128.6, 128.4, 128.1, 128.0, 126.5 (2 × Ph), 80.5 (C-3), 77.4 (C-4a), 68.7 (OCH -Ph), 61.2 (C-8a), 38.6 (C-1'), 30.7 (C-5), 29.6 (C-8), 24.1, 23.7 (C-6, C-7). IR (film): $v=$ 3088, 3064, 3032, 2940, 2863, 1776, 1720, 1498, 1455, 1377, 1333, 1261, 1207, 1146, 1118, 1085, 1030, 800, 752, 737, 699. $[\alpha]^{20}{ }_{\mathrm{D}}=+184\left(c=0.075, \mathrm{CH}_{2} \mathrm{Cl}_{2}\right)$. HRMS (EI, $\left.[\mathrm{M}]^{+}\right): \mathrm{m} / \mathrm{z}$ calcd 379.1784, found 379.1788 .

(2R,1'S,2'S)-2-(2'-Carboxybenzylamino-cyclohexyl)oxy-3-phenyl-propionic acid, Z-COPOH (22). $\mathrm{LiOH} \times \mathrm{H}_{2} \mathrm{O}(7.8 \mathrm{mg}, 0.20 \mathrm{mmol}, 2 \mathrm{eq})$ was dissolved at $0^{\circ} \mathrm{C}$ in $\mathrm{H}_{2} \mathrm{O}_{2}(0.4 \mathrm{~mL}$, 3vol\% in $\left.\mathrm{H}_{2} \mathrm{O}, 0.4 \mathrm{mmol}, 4 \mathrm{eq}\right)$ and added dropwise to a cooled $\left(-18^{\circ} \mathrm{C}\right)$ solution of lactam 21 (36 mg, $95 \mu \mathrm{mol}))$ in THF (2 mL) and warmed to $0^{\circ} \mathrm{C}$ in $1.5 \mathrm{~h} . \mathrm{Na}_{2} \mathrm{SO}_{3}(100 \mathrm{mg})$ was added and the mixture was stirred for $5 \mathrm{~min}$ at $0^{\circ} \mathrm{C}$, acidified to $\mathrm{pH} 1$ with $1 \mathrm{M} \mathrm{NaHSO}_{4}$ and extracted with $\mathrm{CHCl}_{3}(4 \times 10 \mathrm{~mL})$. The combined org. layers were dried $\left(\mathrm{Na}_{2} \mathrm{SO}_{4}\right)$ and concentrated. FCC (3 g, $\mathrm{CH}_{2} \mathrm{Cl}_{2} / \mathrm{MeOH}$ 100:1 $\rightarrow$ 30:1 $\rightarrow$ 10:1) provided acid 22 (25.2 mg, 63.4 $\mu \mathrm{mol}, 67 \%$ ) as a 
colorless gum. TLC: $R_{f}=0.25$ (n-hexane/EtOAc/HOAc 50:50:1). ${ }^{1} \mathrm{H}-\mathrm{NMR}\left(300 \mathrm{MHz}, d_{4^{-}}\right.$ $\mathrm{MeOH}): \delta=0.97-1.30$ (m, 4H, 3'-, 4'-, 5'-, 6'- $\left.\mathrm{H}_{2(\mathrm{ax})}\right), 1.51-1.62$ (m, 2H), 1.65 (m, 1H, 4'-, 5'-, 6'- $\left.\mathrm{H}_{2(\mathrm{eq})}\right), 2.22-2.27$ (m, 1H, 3'- $\left.\mathrm{H}_{2(\mathrm{eq})}\right), 2.82$ (dd, $\left.J=9.1,13.6 \mathrm{~Hz}, 1 \mathrm{H}, 3-\mathrm{H}_{2 \mathrm{~A}}\right), 3.05-3.25$ (m, 3H, 1'-, 2'-H, 3-H $\mathrm{H}_{2 \mathrm{~B}}$ ), 4.15 (dd, $J=3.6,9.4 \mathrm{~Hz}, 1 \mathrm{H}, 2-\mathrm{H}$ ), 5.06 (m, 2H, Z), 7.15-7.39 (m, 10H, $2 \times$ Ph). ${ }^{13} \mathrm{C}-\mathrm{NMR}\left(75 \mathrm{MHz}, d_{4}-\mathrm{MeOH}\right): \delta=158.9$ (Z), 139.8, 138.4, 130.5, 129.4, 129.0, 128.9, 128.8, 127.4, 127.3 (2 × Ph), 80.6 (C-2), 78.6 (C-1'), $67.2\left(\mathrm{O}-\mathrm{CH}_{2}-\mathrm{Ph}\right), 56.7$ (C-2'), 40.6 (C-3), 31.0, 30.9 (C-3', C-6'), 25.1, 24.9 (C-4', C-5'). COOH-carbonyl not detected. HRMS (EI, [M] ${ }^{+}$): $\mathrm{m} / \mathrm{z}$ calcd 397.1889, found 397.1891.

Z-COP-COA-COA-OtBu (23). Dimer 5 (16.4 mg, $40.0 \mu \mathrm{mol}, 1.1 \mathrm{eq})$ was $\mathrm{N}$-terminally deprotected following GP1 and coupled with acid 22 (14.1 mg, $35.5 \mu \mathrm{mol})$ using GP3 (1 mL DMF, $0^{\circ} \mathrm{C} \rightarrow$ r.t., $45 \mathrm{~min}$ ). FCC (3 g, EtOAc/n-hexane 3:1 $\rightarrow$ 3:1 $\rightarrow$ 1:0) provided tripeptide 23 (20.9 mg, $27.4 \mu \mathrm{mol}, 77 \%$ ) as a colorless foam. TLC: $R_{f}=0.21$ ( $n$-hexane/EtOAc 1:2). ${ }^{1} \mathrm{H}$-NMR

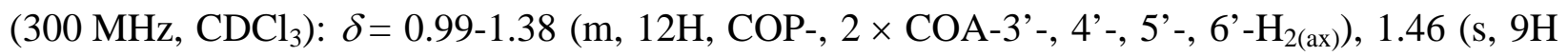
$t \mathrm{Bu}), 1.45-1.77$ (m, 6H, COP-, $2 \times$ COA-4'-, 5'- $\left.\mathrm{H}_{2(\mathrm{eq})}\right), 1.80-2.15$ (m, 5H, COP-6'- $\mathrm{H}_{2(\mathrm{eq})}, 2 \times$ COA-3'-, 6'- $\mathrm{H}_{2(\mathrm{eq})}$ ), 2.23-2.34 (m, 1H, COP-3'- $\left.\mathrm{H}_{2(\mathrm{eq})}\right), 2.73-2.87$ (m, 1H, COP-3- $\left.\mathrm{H}_{2}\right), 2.90-2.99$ (dt, $\left.J=3.4,9.8 \mathrm{~Hz}, 1 \mathrm{H},{ }^{2} \mathrm{COA}-1^{\prime}-\mathrm{H}\right), 3.02-3.22$ (m, 3H, COP-1'-H, 3- $\mathrm{H}_{2},{ }^{3} \mathrm{COA}-1$ '- $\mathrm{H}$ ), 3.373.48 (m, 1H, COP-2'-H), 3.49-3.67 (m, 2H, ${ }^{2-3} \mathrm{COA}-2$ '-H), 3.86 (d, $J=15.0 \mathrm{~Hz}, 1 \mathrm{H}, \mathrm{COA}-2-\mathrm{H}_{2}$ ), $3.94\left(\mathrm{~d}, J=16.9 \mathrm{~Hz}, 1 \mathrm{H}, \mathrm{COA}-2-\mathrm{H}_{2}\right), 4.03\left(\mathrm{~d}, J=15.0 \mathrm{~Hz}, 1 \mathrm{H}, \mathrm{COA}-2-\mathrm{H}_{2}\right), 4.04(\mathrm{~d}, J=$ $16.9 \mathrm{~Hz}, 1 \mathrm{H}, \mathrm{COA}-2-\mathrm{H}_{2}$ ), 4.11 (dd, $J=7.2,7.2 \mathrm{~Hz}, 1 \mathrm{H}, \mathrm{COP}-2-\mathrm{H}$ ), 5.09 (m, 2H, Z), 5.78 (bs, 1H, COP-NH), 7.15-7.39 (m, 10H, $2 \times \mathrm{Ph}), 7.43-7.51$ (m, 2H, COA-NH). ${ }^{13} \mathrm{C}-\mathrm{NMR}(75 \mathrm{MHz}$, $\left.\mathrm{CDCl}_{3}\right): \delta=172.8,171.0,170.4\left({ }^{1} \mathrm{COP}-,{ }^{2-3} \mathrm{COA}-\mathrm{C}-1\right), 156.5$ (Z), 138.1, 136.7, 129.8, 128.5, 128.2, 128.0, 127.9, 126.3 ( $2 \times \mathrm{Ph}), 81.9$ ( ${ }^{2} \mathrm{COA}-\mathrm{C}-1$ ') $, 80.6(t \mathrm{Bu}), 80.4$ ( $\left.{ }^{1} \mathrm{COP}-\mathrm{C}-2\right), 79.8$ ( ${ }^{3} \mathrm{COA}-\mathrm{C}-1$ '), 79.3 ( ${ }^{1} \mathrm{COP}-\mathrm{C}-1$ '), 67.4 ( $\left.{ }^{2} \mathrm{COA}-\mathrm{C}-2\right), 66.5$ (O- $\left.\mathrm{CH}_{2}-\mathrm{Ph}\right), 65.7$ ( $\left.{ }^{3} \mathrm{COA}-\mathrm{C}-2\right), 55.0$ ( ${ }^{1} \mathrm{COP}-\mathrm{C}-2$ ') $)$ 53.4, 53.4 ( ${ }^{2-3} \mathrm{COA}-\mathrm{C}-2$ '), 40.2 ( $\left.{ }^{1} \mathrm{COP}-\mathrm{C}-3\right)$, 31.6, 31.0, 30.9, 30.7, 30.0, 29.9, 29.8 (3× C-3', C-6'), $28.1(t \mathrm{Bu}), 24.1,24.0,23.9,23.7,22.6$ (3× C-4', C-5'). IR (film): $v=3296$, 2933, 2860, 1722, 1666, 1538, 1454, 1339, 1235, 1143, 1122, 846, 752, 699. $[\alpha]^{22}{ }_{\mathrm{D}}=+81.0(c=$ 0.408, $\mathrm{CHCl}_{3}$ ). HRMS (EI, [M] $\left.]^{+}\right): \mathrm{m} / \mathrm{z}$ calcd 763.4408, found 763.4409 . 
Cyclo(-COP-COA-COA-) (24). Tripeptide 23 (20.4 mg, $26.7 \mu \mathrm{mol})$ was consecutively Cterminally deprotected after GP2 $\left(\rightarrow R_{f}=0.23\right.$ in $\left.\mathrm{CH}_{2} \mathrm{Cl}_{2} / \mathrm{MeOH} 10: 1\right)$ and N-terminally deprotected after GP1 (1 h, $\rightarrow R_{f}=0.06$ ). The crude aminoacid was cyclized using GP4 (in pure $\mathrm{CH}_{2} \mathrm{Cl}_{2}$ as solvent). Concentration and FCC (2 g, EtOAc/MeOH 1:0 $\rightarrow$ 100:2 $\rightarrow$ 100:5) provided cyclic tripeptide 24 (12.7 mg, $22.9 \mu \mathrm{mol}, 86 \%)$ as a colorless solid. Single crystals suitable for X-ray crystallography were grown from wet toluene/ $\mathrm{CHCl}_{3}(1: 1)$ by slow evaporation (4 d). Mp 226-228 ${ }^{\circ} \mathrm{C}$. TLC: $R_{f}=0.22$ (EtOAc/MeOH 100:3). ${ }^{1} \mathrm{H}-\mathrm{NMR}$ (300 MHz, $d_{6}$-acetone): $\delta=$ 1.11-1.40 (m, 12H, COP, 2 × COA-3'-, 4'-, 5'-, 6'-H $\left.\mathrm{H}_{2(\mathrm{ax})}\right), 1.56-1.88,2.13-2.19$ (2 × m, 11H, COP-4'-, 5'-, 6'-H 3.56 (m, 6H, COP-3-H2, 1'-, 2'-H, 2 × COA-1'-H), 3.62-3.70 (m, 2H, 2 × COA-2'-H), 3.77 (d, $\left.J=15.1 \mathrm{~Hz}, 1 \mathrm{H}, \mathrm{COA}-2-\mathrm{H}_{2}\right), 3.80$ (d, $\left.J=15.1 \mathrm{~Hz}, 1 \mathrm{H}, \mathrm{COA}-2-\mathrm{H}_{2}\right), 4.01$ (d, $J=15.1 \mathrm{~Hz}, 1 \mathrm{H}$, COA-2-H ${ }_{2}$ ), 4.05 (d, $J=15.1 \mathrm{~Hz}, 1 \mathrm{H}, \mathrm{COA}-2-\mathrm{H}_{2}$ ), 4.21 (t, $\left.J=7.0 \mathrm{~Hz}, 1 \mathrm{H}, \mathrm{COP}-2-\mathrm{H}\right), 7.13-7.23$ (m, 5H, Ph), 7.31 (d, $J=4.5$ Hz, 1H, NH), 7.57 (d, $J=8.1 \mathrm{~Hz}, 1 \mathrm{H}, \mathrm{NH}), 8.10$ (d, $J=6.6 \mathrm{~Hz}, 1 \mathrm{H}$, NH). ${ }^{13} \mathrm{C}-\mathrm{NMR}$ (75 MHz, $d_{6}$-acetone): $\delta=172.8,171.3,170.9$ (COP-, $2 \times$ COA-C-1), 138.6, 130.3, 128.8, 127.0 (Ph), 80.5, 80.3 (2 × COA-C-1'), 79.0, 77.6 (COP-C-2, C-1'), 68.0, 66.9 (2 × COA-C-2), 56.2 (COP-C-2'), 53.7, 53.4 (2 × COA-C-2'), 39.8 (COP-C-3), 31.3, 31.2, 31.1 (3 × C-3’, C-6’), 25.8, 24.9, 24.7, 24.4, 24.3 (3 × C-4', C-5'). IR (film): $v=3372$, 3278, 3083, 3061, 3034, 2934, 2860, 1655, 1535, 1451, 1389, 1341, 1265, 1249, 1141, 1117, 1033, 930, 858, 734, 700. $[\alpha]_{D}^{25}=+151\left(c=0.140, \mathrm{CH}_{2} \mathrm{Cl}_{2}\right)$. HRMS (EI, $\left.[\mathrm{M}]^{+}\right): m / z$ calcd 555.3308, found 555.3306. 


\section{Additional Figures}

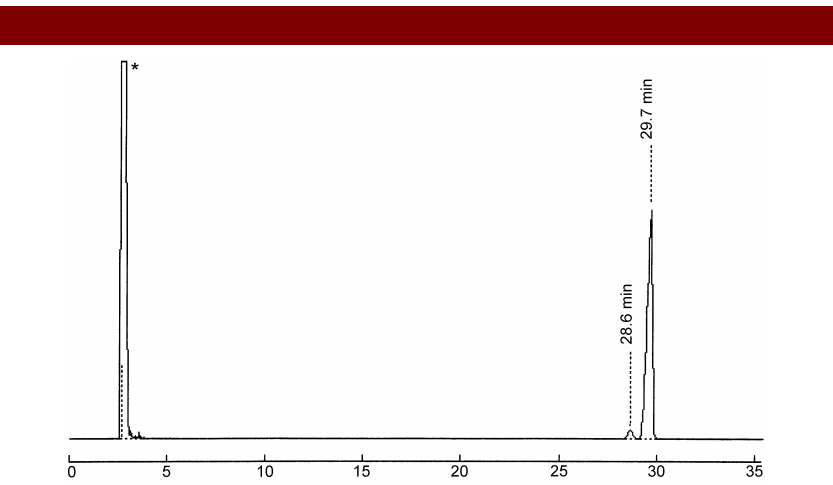

Figure S1. GC trace of azide 1 (50 m FS-Cyclodex alpha -I/P, $T_{\text {col }}=100^{\circ} \mathrm{C}, T_{i n j}=200^{\circ} \mathrm{C}, T_{\text {det }}=250^{\circ} \mathrm{C}$, carrier $\left.\mathrm{H}_{2} 1.0 \mathrm{~kg} / \mathrm{cm}^{2}\right)$. * = Injection peak ( $n$-hexane)

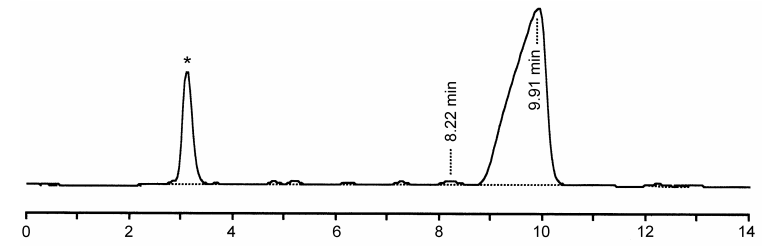

Figure S2. HPLC trace of lactam 21 (Supersphere Si60, n-hexane/i-PrOH 99:1, $1 \mathrm{~mL} / \mathrm{min}, 220 \mathrm{~nm}$ ). * = Injection peak $\left(\mathrm{CDCl}_{3}\right)$.

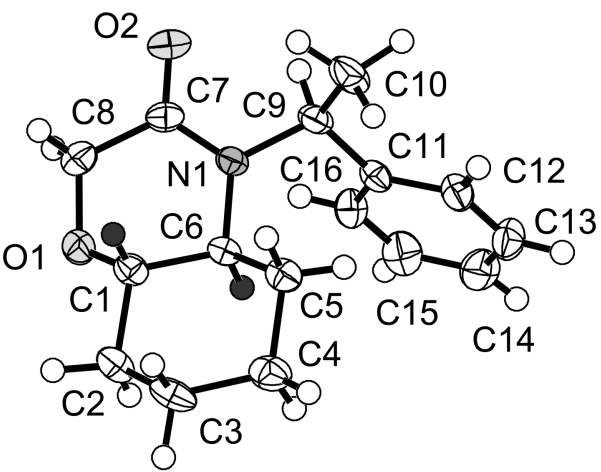

Figure S3. Molecular structure of lactam 18 in the crystal proving its stereochemistry. The trans-configured hydrogen atoms at the ring junctions are marked in dark gray. 


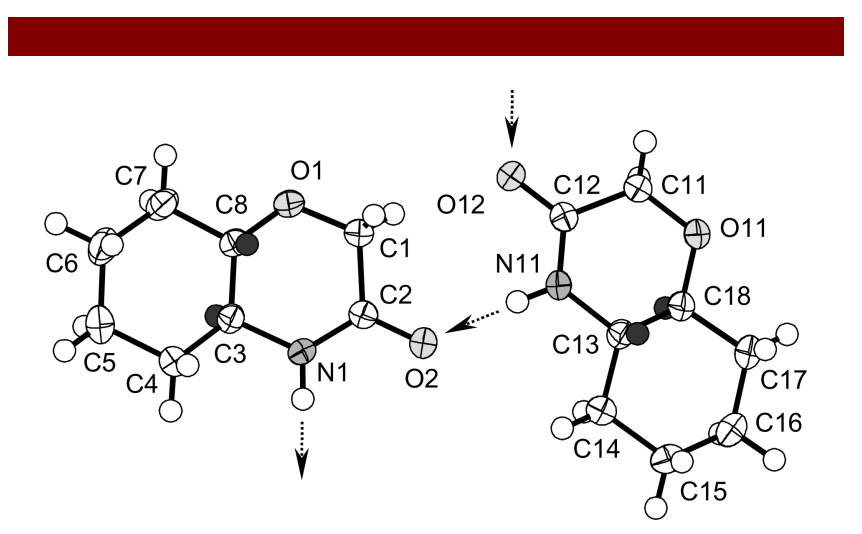

Figure S4. Lactam 19 in the crystal (asymmetric unit). Please note the zig-zag array formed by association of the lactam amides. The ether oxygens do not accept H-bonds.

\section{References}

[1] Arndt, H.-D.; Knoll, A.; Koert, U. Angew. Chem. Int. Ed. 2001, 40, 2076-2078.

[2] Kofron, W. G.; Baclawski, L. M. J. Org. Chem. 1976, 41, 1879-1880.

[3] Gottlieb, H. E.; Kotlyar, V.; Nudelman, A. J. Org. Chem. 1997, 62, 7512-7515.

[4] Martínez, L. E.; Leighton, J. L.; Carsten, D. H.; Jacobsen, E. N. J. Am. Chem. Soc. 1995, 117, 5897-5898.

[5] Leighton, J. L.; Jacobsen, E. N. J. Org. Chem. 1996, 61, 389-390.

[6] de Parrodi, C. A.; Juaristi, E.; Quintero, L.; Clara-Sosa, A. Tetrahedron Asym. 1997, 8, 10751082. 